\title{
Effects of Femtocell Ultradense Deployment on Downlink Performance in LTE Heterogeneous Networks
}

\author{
Motea Saleh Al-omari, ${ }^{1}$ Mohammad Ahmed Alomari $\mathbb{D}^{2}{ }^{2}$ Abdul Rahman Ramli, ${ }^{1}$ \\ Aduwati Sali, ${ }^{1}$ Raja Syamsul Azmir, ${ }^{1}$ and M. Hafiz Yusoff $^{3}$ \\ ${ }^{1}$ Faculty of Engineering, Universiti Putra Malaysia, Selangor, Malaysia \\ ${ }^{2}$ Institute of Informatics and Computing in Energy, Universiti Tenaga Nasional, Malaysia \\ ${ }^{3}$ School of Informatics and Computing, Albukhary International University (AIU), Malaysia
}

Correspondence should be addressed to Mohammad Ahmed Alomari; engr_omari@yahoo.com

Received 18 December 2019; Revised 19 April 2021; Accepted 7 August 2021; Published 10 September 2021

Academic Editor: Vicente Casares-Giner

Copyright ( 2021 Motea Saleh Al-omari et al. This is an open access article distributed under the Creative Commons Attribution License, which permits unrestricted use, distribution, and reproduction in any medium, provided the original work is properly cited.

\begin{abstract}
With huge number of smart gadgets and wireless devices being interconnected to each other, the demand for very high data bandwidth is becoming critically challenging. With such density of nodes inside wireless networks, providing high-quality service as well as wide coverage in indoor environment is a real challenge, which is due to the limited radio frequency and intense interference between nodes. As a one way to solve such problem and improve indoor service quality, femtocells have been introduced as an extension to the existing macrocell stations. Although femtocell is a promising technology, the pervasive deployment of huge number of femtocells without very tight network planning as well as coverage strategy may worsen the problem and degrade the service quality. One important problem that needs to be addressed when deploying femtocell technology in heterogenous networks (HetNets) is mitigating the various types of cross-tier and cotier interferences in between wireless cells. This study investigates the effect of unplanned ultradensity femtocell deployment in the downlink performance of two-tier heterogeneous networks in urban area based on LTE system. Instead of deploying femtocells one by one, grids of size either $(3 \times 3)$ or $(5 \times 5)$ of neighboring femtocell will be deployed inside each macrocell sector area. The simulation results show that femtocell deployment improves overall average user throughput in case of low and medium density scenarios. However, for ultradensity scenario, there is no enhancement in terms of fairness and throughput. The results confirm that this leads to high degradation for macrocell and femtocell user performance due to the severe interference between macrocells and femtocells, as well as among neighboring femtocells in each grid.
\end{abstract}

\section{Introduction}

Due to the advances in modern cellular technology, wireless devices have become an essential indispensable element in our daily life. The rapid growth and proliferation of wireless devices and gadgets have turned the cellular networks from being basically voice networks to become mostly data networks. To provide higher data rates, operators across the world have switched to upper frequencies in order to increase the bandwidth. However, increasing number of wireless nodes in suburban and urban areas has created a bigger challenge for the outdoor base station to provide quality coverage in indoor environments. According to recent studies, roughly $66 \%$ of the phone calls and $90 \%$ of the data services take place in indoor environments (e.g., home, office, and enterprises) [1-3]. However, more and more users complain of poor connectivity as the signal strength received from outdoor base station (BS) weakens and deceases in performance due to the high loss of wall penetration [4-6]. As stated in [7], 45\% of the residential subscribers and $30 \%$ of the corporate subscribers complain of poor indoor coverage. The existing outdoor base station cannot increase the transmission power in an infinite level to ensure the coverage in outage areas. The growing demand for broadband wireless access inside residential (indoors) environment along with the shortage of capacity in the 
existing outdoor base stations has motivated wireless service providers to look for possible solutions to improve indoor coverage with high data rates and enhanced quality of service (QoS). To handle these challenges, different deployment strategies have been proposed earlier including installation of extra macrocell, distributed antennas (DAs), hot spots, in-building solution (IBS), picocells, and multihop relays. The infrastructure for doing so, nonetheless, is expensive and cannot guarantee high quality indoor coverage. The concept of femtocells has recently been introduced as the cheapest solution for indoor wireless broadband [8-10]. Femtocell base station (FBS) or femtocell access point (FAP) is a small base station typically designed for indoor use in a small office home office (SOHO) environment to provide voice and broadband services. FBS is a cost competitive, low power, short-range wireless device operating in the licensed spectrum. The term femtocell itself was first coined in 2006. FBS is not a new concept as it was first studied in 1999 by Bell Labs, while GSM-based home base station was brought to the market by Alcatel in 2000. The first 3G-based home base station was introduced by Motorola in 2002 [11]. In the metric measurement system, "Femto" means one-quadrillionth $\left(10^{15}\right)$. FBS is installed by the users to ensure seamless indoor coverage with better voice and data reception $[12,13]$. FBS, on the air interface, provides radio coverage to a given cellular standard [14], such as Global System Mobile (GSM) communication, Wideband Code Division Multiple Access (WCDMA), Universal Mobile Telecommunications System (UMTS), Worldwide Interoperability for Microwave Access (WiMAX), and Long-Term Evolution (LTE). The 3rd Generation Partnership Project (3GPP) refers to this FBS as Home Node Base station (HNBs). The LTE femtocell is referred to as Home evolved Node Base station (HeNB) [15]. As illustrated in Figure 1, FBSs are connected to the Internet via broadband backhaul connection such as cable modem, digital subscriber line (DSL), on premise fiber optic link, separate radio frequency (RF) backhaul channel, satellite, or a similar IP backhaul technology. The wired connection is used to integrate the FBS with the mobile operator's core network. FBSs were introduced in the 3GPP release 8 in 2008 [16].

Given the continuous evolving of technologies of cellular networks, several femtocell specifications and standards are being included in the technical reports of 3GPP and 3GPP2 [14, 17, 18]. Triggered by its high spectral efficiency and low implementation cost, OFDMA has been utilized in many high-speed wireless transmission standards (e.g., LTE, WiMAX, and Wi-Fi). Long-Term Evolution (LTE) is a broadband wireless access technology designed to support mobile Internet access via cell phones or handheld devices. As LTE exhibits significant performance improvements over previous cellular communication standards, it is commonly referred to as fourth generation (4G) technology [19]. This paper treats LTE as a standard to investigate the interference problem in OFDMA-based femtocell networks. The standard was developed by the 3GPP and was specified and enhanced in release 8 and release 9 of its document series, respectively [20]. Besides LTE, LTE-advanced has been developed as successor standard of LTE. There are many solutions which have been specified in release 10 and release 11 (LTE-advanced) of LTE, and more solutions are studied and developed in the next release (Rel-12 and beyond). A number of worldwide mobile operators showed interest in LTE HeNB technologies as a promising solution to increase system capacity in the near future $[21,22]$.

This paper is intended to focus on investigating the challenges and impact of unplanned (random) femtocell deployment density on downlink performance of MUE (macrouser equipment) in LTE heterogeneous networks. The rest of the paper moves as per the following roadmap; Section 2 provides various indoor coverage technologies in heterogeneous networks. Section 3 discusses in detail the interference issue and provides different interference scenarios in OFDMA two-tier macrofemtocell heterogeneous network-based LTE. Section 4 describes the system model and the effects of deployment FBS grid density in HetNets. Section 5 provides the performance evaluation metrics while Section 6 provides the simulation environment and lists all simulation parameters. Finally, Section 7 presents the simulation results while Section 8 provides concluding remarks.

\section{Indoor Coverage Technologies in HetNets}

The traditional cellular network deployment approach exhibits to only macrocentric cell architecture where the macrocell base stations correspond to a planned layout. Such macrocell-based cellular network is referred as homogeneous network. In homogeneous network, all the base stations have similar backhaul connectivity to the core network, same transmit power levels, antenna heights, antenna patterns, and receiver noise floors, and provide unrestricted connectivity to all the user equipments (UEs) [22, 23]. A network which includes the hierarchical layering of cells with macro base stations coexisting with low-power and short-range small cells (such as femtocells, picocells, distributed antenna systems (DASs), and relays) in the same service area is referred to as a Heterogeneous Networks (HetNets) or a multitier network as illustrated in Figure 2. HetNets or a multitier network is considered to be an efficient solution to enhance the spectral-efficiency per unit area of the network. Additionally, it also provides significant improvement in the coverage of the indoor and cell-edge users as well as assuring better quality of service (QoS) to various users. In recent years, HetNet-based deployment model is considered by the mobile operators. In other words, HetNets or multitiered cellular network comprises of existing homogeneous networks overlaid with extended infrastructure in the form of smaller, low-complexity, low-power, and short-ranged base stations referred as femtocells (small cells) [24].

The radio base stations in HetNets can contain various types such as macrocells, picocells, and relay nodes. Macrocell base stations, which is referred as MeNBs, have the ability to cover larger area (e.g., cell radius of $500 \mathrm{~m}-1000 \mathrm{~m}$ ) with high transmit power $(46 \mathrm{dBm}$ or 40 watts) while providing service to all the UEs in its coverage area [25]. The abbreviations MBS, MC, and MeNBs are all stands for macro base station and may be used interchangeably. 


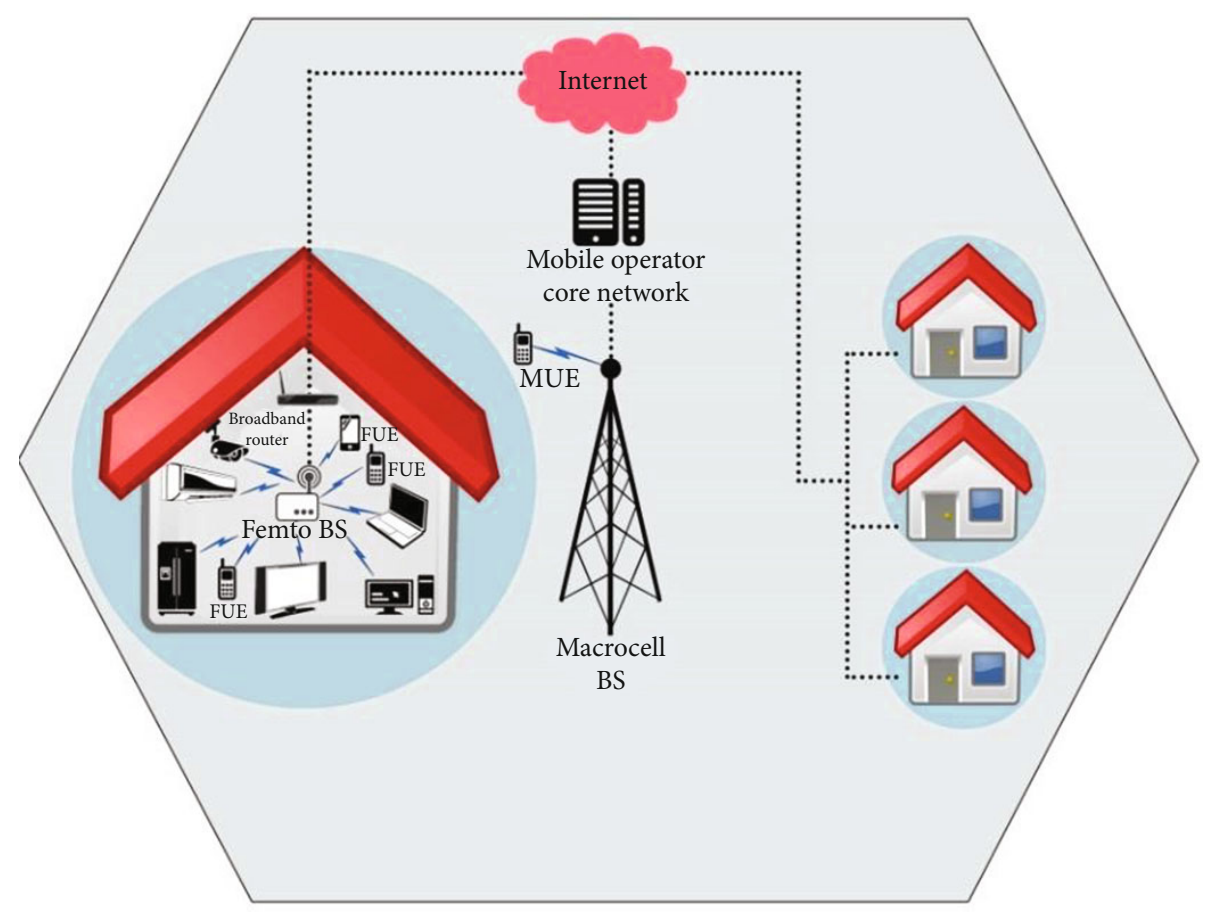

FIgURE 1: A typical femtocell architecture.

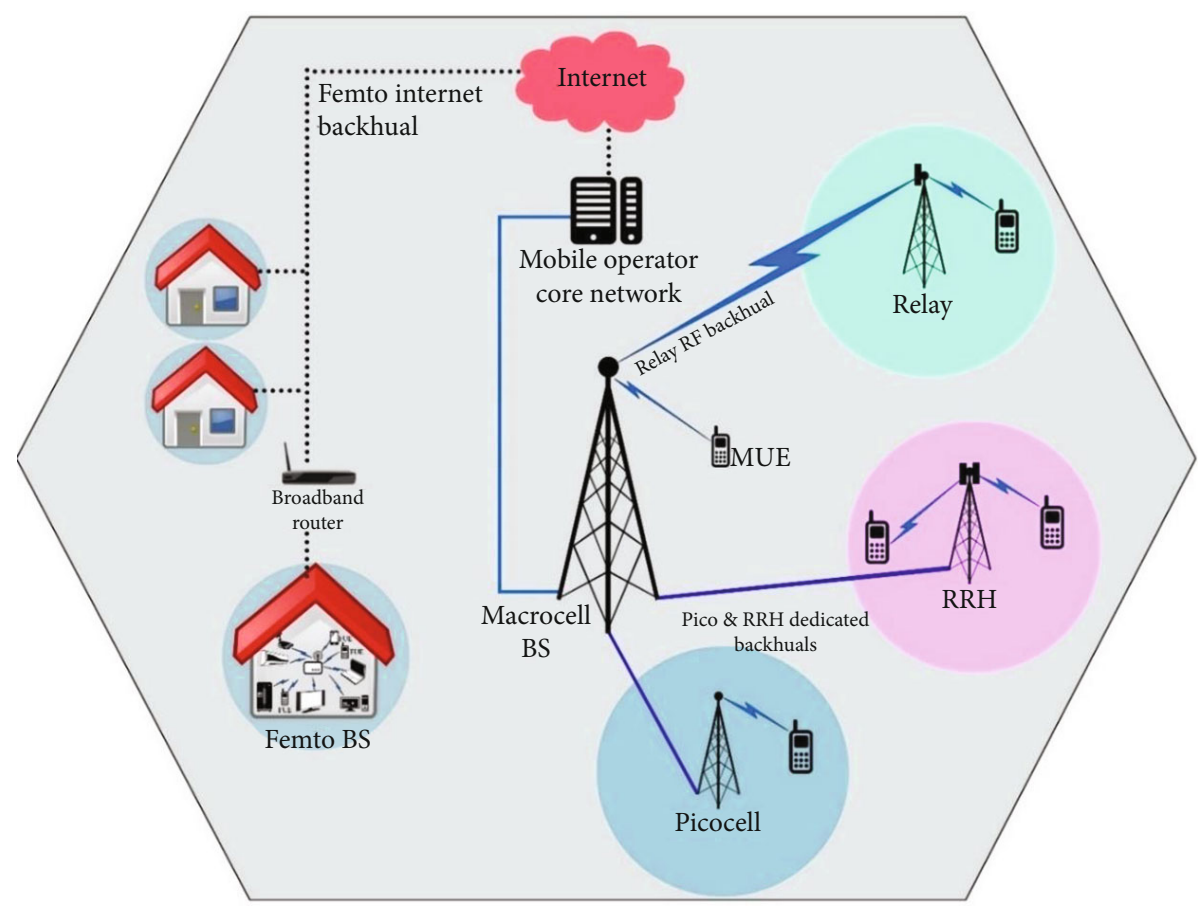

FIgURE 2: Layout of a heterogeneous cellular wireless network concept.

Picocells are smaller wireless towers which are usually deployed to minimize coverage holes in a homogeneous cellular network. They help improving the capacity of the network. The coverage area of a picocell usually can span between $75 \mathrm{~m}$ and $150 \mathrm{~m}$. In comparison to MeNB, the picocells consist of omni-directional low-transmission powered antennas with providing proper indoor coverage to the UEs in the public places such as shopping malls, stadiums, and airports [26]. DASs comprise of several separate antenna elements (AEs) connecting to macro base stations (MBSs) via dedicated radio frequency (RF) links or fiber optic cables to extend the macrocoverage. Like picocells, 
TABLE 1: Comparison of various indoor wireless access technologies.

\begin{tabular}{|c|c|c|c|c|c|}
\hline Specifications & Femtocell & Picocell & DAS & Relay & $\mathrm{Wi}-\mathrm{Fi}$ \\
\hline Spectrum type & Licensed & Licensed & Licensed & Licensed & Unlicensed \\
\hline Typical power & $10-100 \mathrm{~mW}$ & $\begin{array}{l}250 \mathrm{~mW}-2 \mathrm{~W} \text { (out), } \\
<100 \mathrm{~mW} \text { (indoor) }\end{array}$ & & $\begin{array}{c}250 \mathrm{~mW}-2 \mathrm{~W} \text { (out), < } \\
100 \mathrm{~mW} \text { (indoor) }\end{array}$ & $100-200 \mathrm{~mW}$ \\
\hline Power control & Support & Support & Support & Support & Not support \\
\hline Coverage range & $20-50 \mathrm{~m}$ & $150 \mathrm{~m}$ & Macrocoverage extension & Macrocoverage extension & $100-200 \mathrm{~m}$ \\
\hline Services & Voice and data & Voice and data & Voice and data & Voice and data & Data and VOIP \\
\hline Handset device & $\begin{array}{l}\text { All ordinary } \\
\text { devices }\end{array}$ & All ordinary devices & All ordinary devices & All ordinary devices & $\begin{array}{l}\text { Dual mode } \\
\text { devices }\end{array}$ \\
\hline $\begin{array}{l}\text { Working } \\
\text { environment }\end{array}$ & $\begin{array}{l}\text { Indoor (home/ } \\
\text { office) }\end{array}$ & $\begin{array}{l}\text { Outdoor/indoor (hot spot/ } \\
\text { office) }\end{array}$ & Indoor extension & $\begin{array}{l}\text { Hot spot/office/tunnel, high } \\
\text { speed train }\end{array}$ & $\begin{array}{l}\text { Indoor/outdoor } \\
\text { Home/hot spot }\end{array}$ \\
\hline Backhaul & $\begin{array}{l}\text { DSL/cable/ } \\
\text { optical fiber }\end{array}$ & $\mathrm{X} 2$ interface & $\begin{array}{l}\text { Optical fiber or RF links } \\
\text { to macrocell }\end{array}$ & $\begin{array}{l}\text { Wireless in-band or out-of- } \\
\text { band }\end{array}$ & $\begin{array}{l}\text { DSL/cable/ } \\
\text { optical fiber }\end{array}$ \\
\hline $\begin{array}{l}\text { Access } \\
\text { methods }\end{array}$ & $\begin{array}{l}\text { Closed/open/ } \\
\text { hybrid }\end{array}$ & Open access & Open access & Open access & Closed/open \\
\hline Security & Robust & Robust & Robust & Medium & Fades \\
\hline Installation & By consumer & Operator & Operator & Operator & Consumer \\
\hline Data rate peak & \multicolumn{4}{|c|}{ LTE-advanced (3GPP R10): $1 \mathrm{G} \mathrm{b/s} \mathrm{(DL)} 300 \mathrm{M} \mathrm{b/s(UL)}$} & $\begin{array}{c}600 \mathrm{Mb} / \mathrm{s} \\
(802.11 \mathrm{n})\end{array}$ \\
\hline
\end{tabular}

Table 2: Femtocell versus Wi-Fi.

\begin{tabular}{lcc}
\hline Specifications & Femtocell & Wi-Fi \\
\hline Standard & 3GPP, 3GPP2 femtocell standard & IEEE.802.11 a/b/g/n \\
Operating frequency & $1.9-2.6 \mathrm{GHz}$ (licensed) & $2.4-5 \mathrm{GHz}(\mathrm{unlicensed})$ \\
Power & $10-100 \mathrm{~mW}$ & $100-200 \mathrm{~mW}$ \\
Coverage range & $20-50 \mathrm{~m}$ & $100-200 \mathrm{~m}$ \\
Data rates & $7.2,14.4,100 \mathrm{Mbps}$ & $11,54,500 \mathrm{Mbps}$ \\
Services & Voice and data & Data and voice \\
Handset & All ordinary phone devices & Dual mode devices \\
Complexity of service & Relatively low & High \\
Signal robustness & Robust & Fades \\
\hline
\end{tabular}

relay nodes are also used to improve service coverage in new areas such as exhibitions, events, tunnels, or high-speed trains. However, relay nodes backhaul their data traffic through wireless link to a donor eNodeB (e.g., MeNB).

Various technologies for indoor coverage are summarized in Table 1. Wi-Fi router in wireless local area networks (WLAN) is a plug and play broadband wireless access point technology. Wi-Fi access point is similar to FBS in terms of size and providing fixed mobile convergence (FMC) [24]. Both FBS and Wi-Fi are backhauled to the Internet via a broadband connection. Therefore, their performance is affected by IP network conditions. The FBS implements cellular technology which can initiate a call in a real time using licensed spectrum while Wi-Fi WLAN mainly used for data services and voice over IP (VoIP) using unlicensed spectrum. However, femtocell and Wi-Fi are considered as competitive technologies. Table 2 provides a summarized comparison between femtocell and Wi-Fi technologies [24].

\section{Interference Issues in OFDMA Macrofemto LTE HetNets}

In wireless broadband access networks, most indoor environments encounter serious coverage problem due to nonline of sight transmission. Femtocells have been introduced as cost efficient solution to improve cell coverage, enhance area spectral-efficiency, and provide better quality of service (QoS) to mobile users. However, vast deployment of femtocell network in the absence of proper network planning strategy makes it difficult to maintain the desired quality of service. Additionally, the service of femtocell in densely deployed heterogeneous network is challenged by interference $[27,28]$. In reality, interference mainly occurs due to the fact that the radio resource is scarce. Femtocells have to share the available spectrum resource (use the same licensed frequency band) with the existing macrocells in the network. This process is referred to as cochannel 


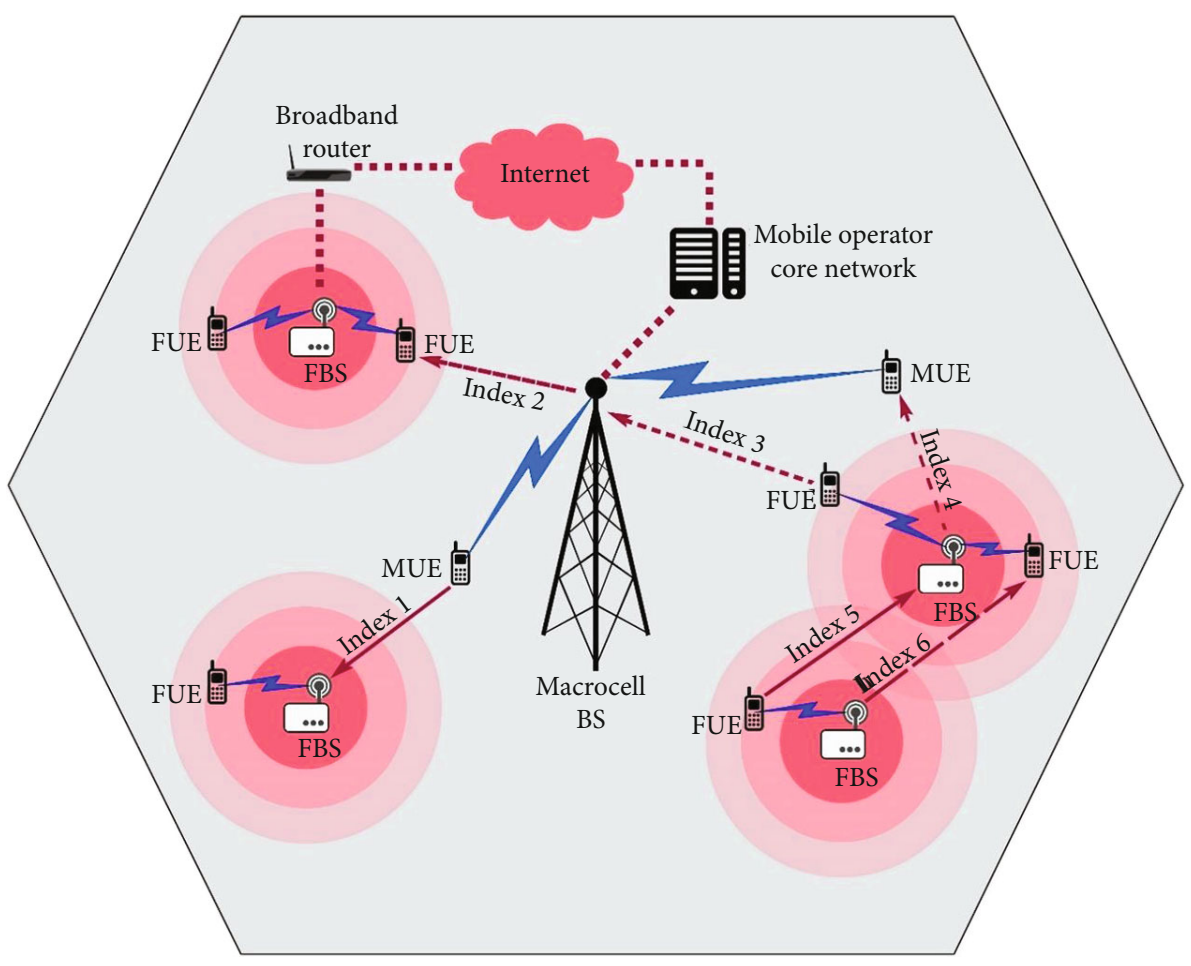

Figure 3: Interference scenarios in two-tier macrocell and femtocell HetNets [32].

deployment which may cause severe interferences between femtocells and macrocells [29]. One drawback of femtocells is that its coverage will extend outside of a home to the neighboring premises causing interference among nearby femtocells. Moreover, adding new femtocells may cause operational disruption to existing femtocells. Due to their easy installation, femtocell base stations can be installed by end users or less experienced operators which make managing the interference more difficult and highly degrade system performance. Such interference can impact not only femtocell performance, but it may also severely affect the performance of other devices connected to macrocell network leading at the end to "Dead Zones" $[30,31]$. A dead zone occurs when the communication link can not be established due to the SNIR (signal-to-interference-plus-noise ratio) reading falls below a particular threshold.

Generally, interference in two-tier macro and femtocell HetNets can classified into two types: cross-tier interference and cotier interference.

3.1. Cross-Tier Interference or (Intertier Interference). Crosstier interference will result due to the interference between elements that belong to different tiers of the network. As an example is the interference occurring between macrocell elements and femtocell elements. Here, the macrocell UEs (known as MUEs) and femtocell UEs (FUEs) are the sources of uplink cross-tier interferences towards the nearby FBSs as well as the serving macrocell base station (MBS), respectively [30], as illustrated with index 1 and index 3 in both Figure 3 and Table 3. Conversely, the serving MBS, together with the FBS, results in forward link (downlink) cross-tier interferences on to the FUEs and the neighboring MUEs as illus-
TABLE 3: Interference scenarios for two-tier macrocell and femtocell HetNets [32].

\begin{tabular}{lcccc}
\hline Index & Interference type & Aggressor & Victim & Transmission mode \\
\hline 1 & Cross-tier & MUE & FBS & UL \\
2 & Cross-tier & MBS & FUE & DLk \\
3 & Cross-tier & FUE & MBS & UL \\
4 & Cross-tier & FBS & MUE & DLk \\
5 & Cotier & FUE & FBS & UL \\
6 & Cotier & FBS & FBS & DL \\
\hline
\end{tabular}

trated with indexes 2 and 4, respectively, shown in both Figure 3 and Table 3. Figure 3 provides a summary of the various cross-tier scenarios. Nevertheless, for OFDMA networks, cross-tier forward (DL)/reverse link (UL) interferences happen exclusively in cases where both the aggressor and the victim attempted to use the same radio spectrum resources (subchannels, resource blocks) [32-34]. Thus, efficient resource allocation is indeed vital for interference avoidance is such systems.

3.2. Cotier Interference (Intratier Interference). As the name suggests, cotier interference, in general, is the interference which takes place between network elements belonging to the same tier. In terms of femtocell networks, cotier interference refers to the interference among neighboring femtocells. Cotier interference occurs in two different forms uplink cotier interference and downlink cotier interference $[5,35]$. The former occurred when femtocell user equipment FUE (aggressor) may cause uplink cotier interferences with its neighboring FBSs (victim) as illustrated with index 5 in 
both Figure 2 and Table 3. The latter interference occurred when an FBS (aggressor) may also cause downlink cotier interferences to a nearby FUEs (victims) as illustrated with index 6 shown in both Figure 1 and Table 3. For femtocell networks based on OFDMA, the occurrence of cotier uplink (UL)/downlink (DL) interferences takes place only when both the aggressor and the victim attempt to communicate using the same spectrum resources (subchannels or resource blocks). Thus, for interference mitigation in such networks, an intelligent and efficient radio resource allocation mechanism is required.

In orthogonal frequency division multiple access(OFDMA-) based macrocellular long-term evolution (LTE) femtocell networks, (uplink/downlink) interferences occur only when the aggressor and the victim attempted to share and/or reuse the same radio frequency resource (subchannels, resource blocks). Since the radio spectrum (frequency) resources are limited, then the resource allocation considers an important factor in terms of interference avoidance in two-tier HetNets [36-38]. Therefore, by adopting suitable resource allocation, the cross-tier interferences can be considerably minimized, while the cotier interferences can be effectively avoided, and this will lead to improve the overall system performance.

Table 3 gives a brief description on interference types (cross-tier, cotier), source (aggressive), victim of interference, and transmission mode (UL, DL) for various interference scenarios that depicted in Figure 1 and indexed from 1 to 6 .

\section{System Design Model and Effects of Deploying FBS Grid Density in HetNets (DL)}

Femtocell is deployed and installed randomly by users without any prior planning from the operator. However, femtocell deployment is assumed either normal distribution or specific location in most of previous studies [39, 40]. Such femtocell deployment is not simulated the real situation especially for urban (enterprise with high building or highrise residential condominiums). Therefore, the future is supposed to consider the most realistic or the worst scenario for femtocell deployment. Moreover, large scale femtocell deployment, especially when femtocell density increases dramatically, still needs more research efforts [24]. This paper considers the worst scenario for femtocell deployment, to investigate the impact of high-density femtocell deployment on downlink performance of MUEs in LTE heterogeneous. In the proposed model, femtocell density dramatically increases because of large scale femtocell deployment as grids of either size $(3 \times 3)$ or size $(5 \times 5)$. In addition, such deployment provides the worst scenario in terms of cotier interference among neighboring femtocells.

In this section, system model assumes two-tier macro and femtocell HetNets. Figure 4 depicts a system model hexagonal-grid macrocell scenario with seven BSs and 21 sectors. This scenario is used in simulations throughout the paper. The coverage of each macrocell can classify into two-types: outdoor and indoor area. The outdoor area is covered by macro BS whereas the indoor area is covered either by FBS or macro BS. The applied vertical/horizontal antenna radiation pattern corresponding to realistic antenna is supplied by KATHEREIN-Werke KG according to a 742215-panel antenna.

This model assumes that macro base stations (MBS) and femto base stations (FBS) working in an autonomously manner (i.e., there is no coordination between them and employ universal frequency reuse (reuse-1) in terms of their transmission). The resource allocation uses proportional fair scheduling in order to ensure fairness for users of both macrocell and femtocell [41].

4.1. Macrocell Geometry. A hexagonal layout is considered for each macrocell site. The model composed of seven hexagonal macrocells labeled from BS0 to BS6 site separated by distance $d$ (BSO is the label for macrocell site number $0)$. In addition, each macrocell site is holding three hexagonal sectors separated by $120^{\circ}$ and equipped with three BSs, each one employing a tilted directional antenna with $15 \mathrm{~dB}$ maximum antenna gain, as depicted in Figures 5(a) and 5(b), respectively.

4.2. User Distribution. Users are randomly distributed in each macrocell sector according to a Poisson Cluster Process [42]. The label $U(x, y)$ in Figure 5(a) shows that a user positioned at $(x, y)$ coordination. MUEs distributed randomly in each sector-site and according user's positions $(x, y)$; the user will be considered as outdoor or indoor macrouser. A set of femtocell users (FUEs) are uniformly distributed within each indoor area of FBS. The FUE only located within the indoor coverage of FBS; otherwise, it will be handoff to MBS and on that time will consider as MUE. This study assumes the wall isolate indoor environment from the outdoor area, which is modeled as a penetration loss.

4.3. Femtocell Base Station. FBSs are equipped with omnidirectional antennas of $0 \mathrm{~dB}$ gain. In this model, the femtocells assumed to be randomly distributed inside each macro hexagonal-sector site as $(3 \times 3)$ or $(5 \times 5)$ grids of neighboring houses. Each square of houses, which combinly forms the grid, will have a dimension of $10 \times 10 \mathrm{~m}$. The FBS is installed by users themselves and randomly placed inside the house. This is reflecting the fact that femtocell deployments related to the unplanned part of the network and then interference issue become a big challenge. The coverage area of each femtocell is illustrated as circularly shaped of radius $(R)$. The $(3 \times 3)$ or $(5 \times 5)$ femtocell grids are spreading randomly over a macrocell BS's sector site coverage area. In this model, the total number of neighboring deployed femtocells per grid is either 9 femtocells as in $(3 \times 3)$ grid or 25 femtocells as in $(5 \times 5)$ grid as illustrated in both Figures $6(\mathrm{a})$ and 7 (a), respectively. In the simulations, the femtocell grids will add to each macrocell network sector area as FBS grid deployments as shown in details models below.

4.3.1. Deployment of Femtocell as Grids $(3 \times 3)$. In this type, the femtocells deploy as a block and/or grid of squares. Figure 6(a) shows the model of such deployment. Each block represents a grid of $3 \times 3$ squares for femtocell deployments on each macrocell sector area. Each square from the grid 


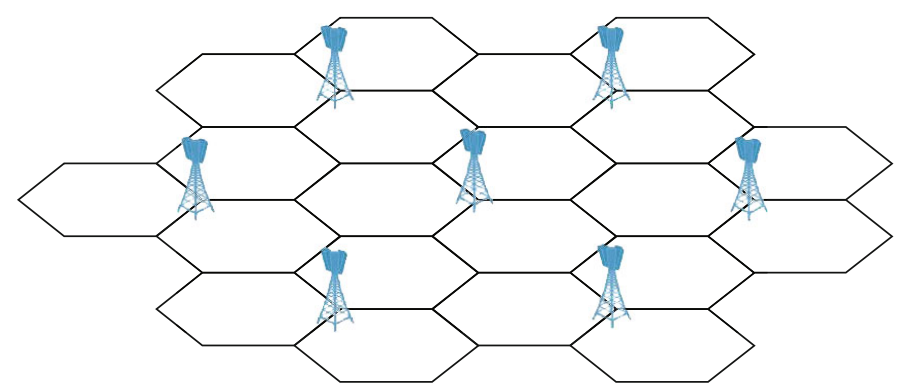

FIGURE 4: Hexagonal-grid macrocell scenario with seven BSs and 21 sectors.

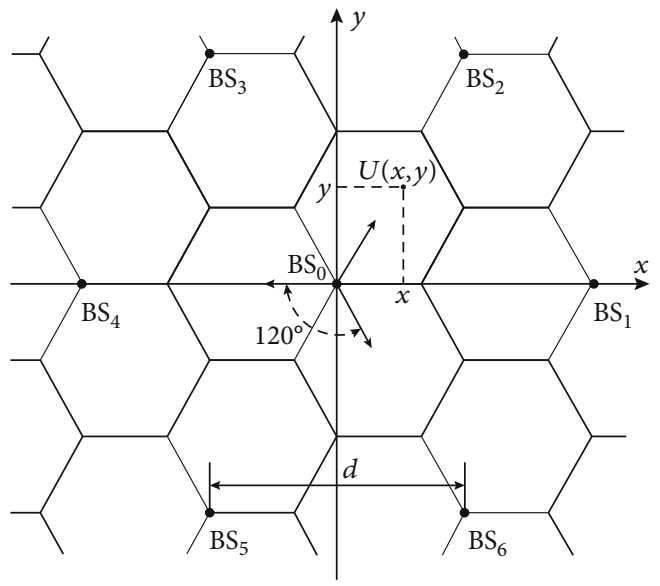

(a)

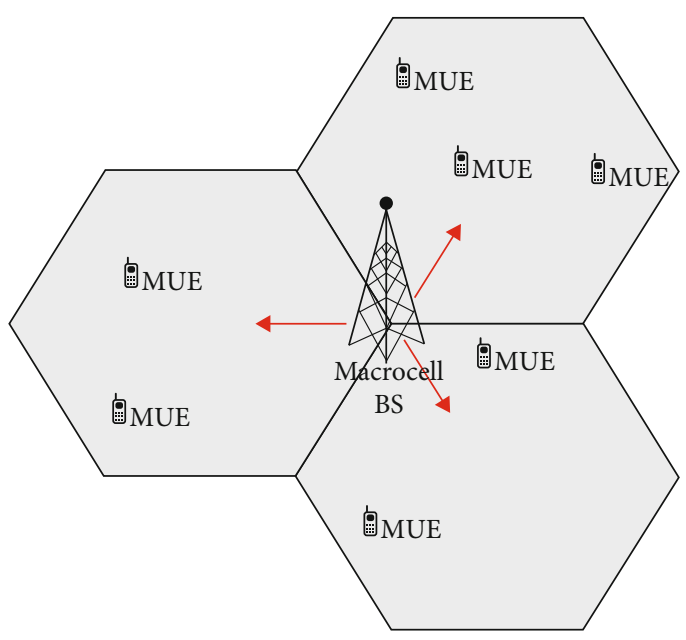

(b)

Figure 5: Outdoor and indoor user distribution per macrocell area. (a) 7-macrocell site with three hexagonal sectors for each. (b) A macrocell geometry with three hexagonal sectors separated 120 .

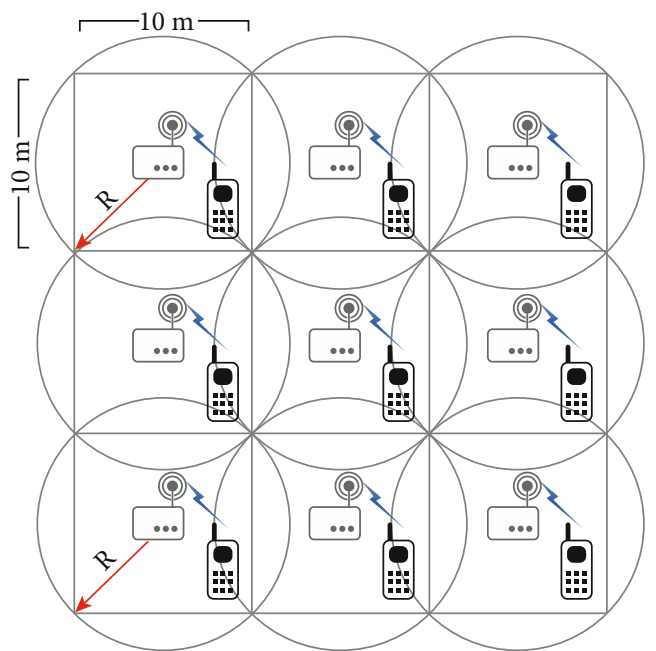

(a)

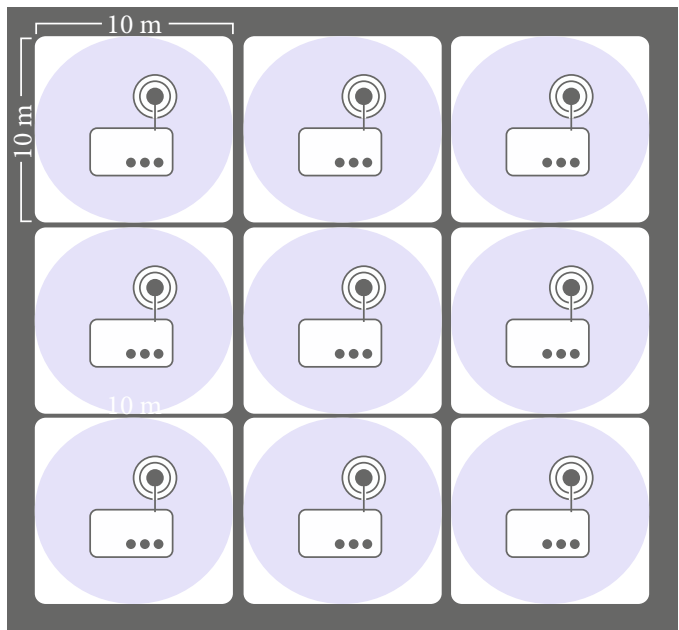

(b)

Figure 6: (a) FBS grid size $(3 \times 3)$. (b) Cotier interference in FBS grid $(3 \times 3)$.

represents a house and having dimension of $10 \times 10$ meter. This scenario is similar to the model proposed by $3 \mathrm{GPP}$ which modeled as two stripes of apartments; each stripe has 2 by $N$ apartments. Each apartment is of size $(10 \times 10)$ meter. There is a street between the two stripes of apartments, with width of $10 \mathrm{~m} \mathrm{[4].} \mathrm{However,} \mathrm{the} \mathrm{proposed} \mathrm{model}$ is considering more realistic because the FBS assumes to be randomly placed inside the square, so that a total of 9 


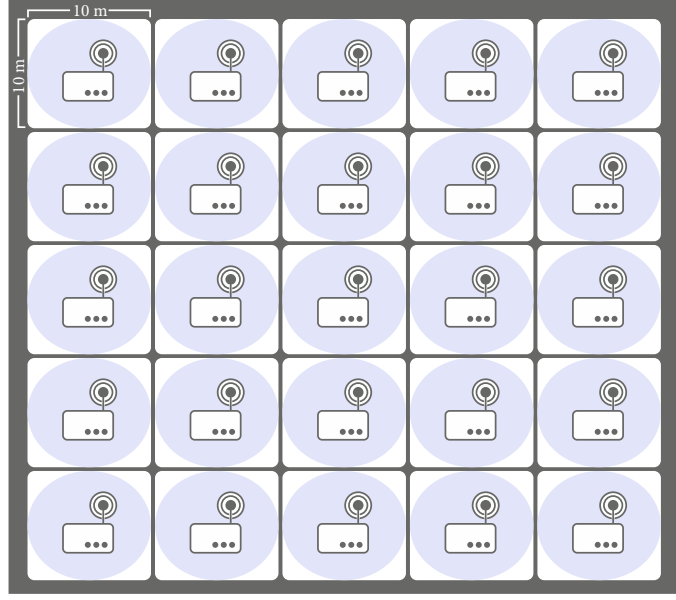

(a)

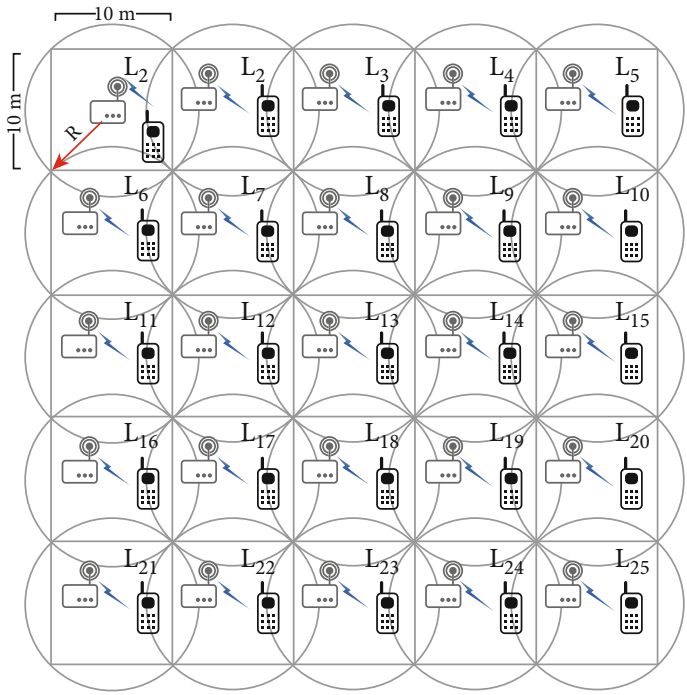

(b)

Figure 7: (a) FBS grid size $(5 \times 5)$. (b) Cotier interference in FBS grid $(5 \times 5)$.

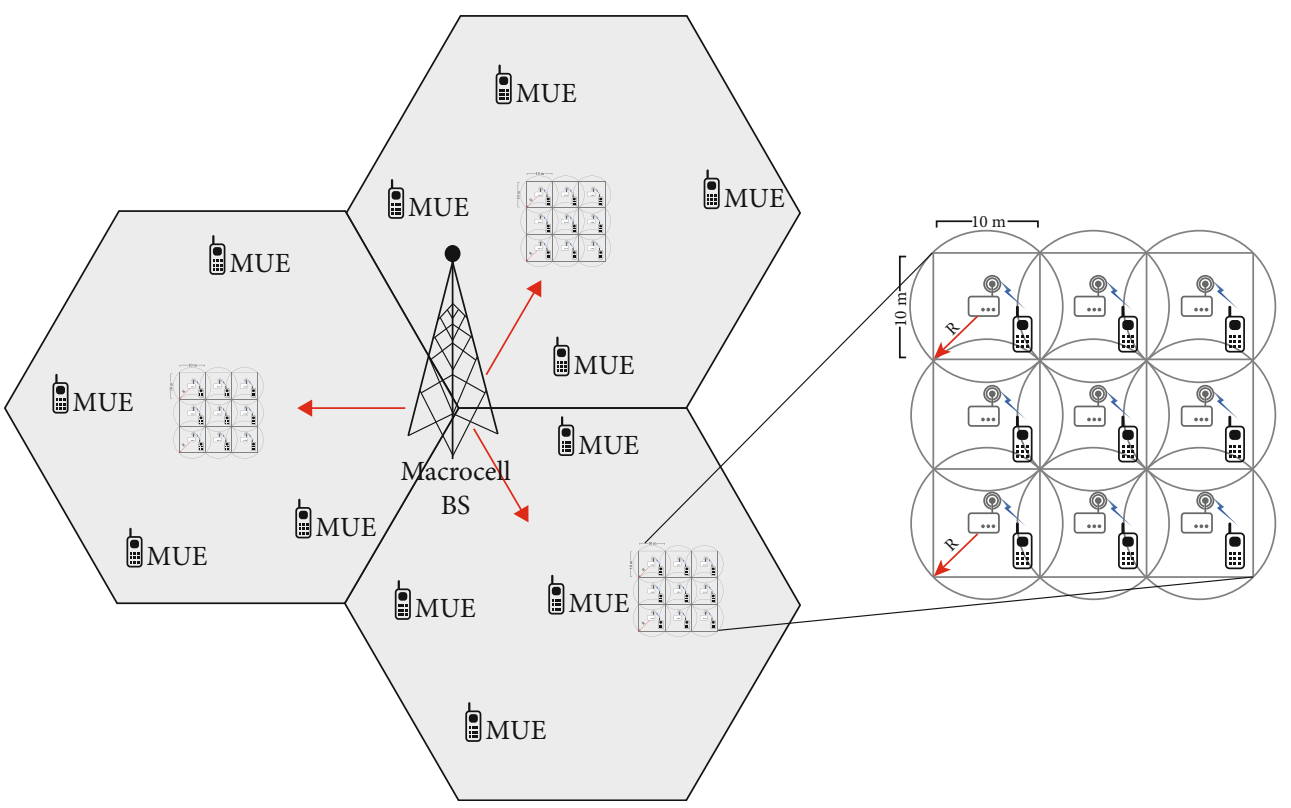

Figure 8: Two-tier HetNets for macrocell overlaid with femtocell grids $(3 \times 3)$.

neighboring femtocells deployment per grid. Figure 6(b) shows the worst case for cotier interference among neighboring femtocell in $3 \times 3$ grid block when FBS coverage exceeds to outside of the served square house. Figure 8 shows a two-tier HetNets of macrocell overlaid with $3 \times 3$ femtocell grids per sector area.

4.3.2. Deployment of Femtocells as Grids $(5 \times 5)$. In this scenario, a grid of $5 \times 5$ femtocell deploys on each macrocell sector area. Figure 7 (a) shows the model of such deployment; each square from the grid represents a house with dimension of $(10 \times 10)$ meter. The FBS assumes to be placed randomly inside the square house, so that a total of 25 neigh- boring femtocell deployment per grid. Deployment femtocells as grid of size $(5 \times 5)$ will increase the femtocell density per sector area in a fast manner. Therefore, when the number of $(5 \times 5)$ femtocell grids increased per macrocell area, then the femtocell density becomes so high, and the cotier interference among neighboring femtocells will severely increase as depicted in Figure 7(b). Figure 9 shows a two-tier HetNets of macrocell overlaid with $5 \times 5$ femtocell grids per sector area.

The number of femtocell grids per macrocell BS sector area is denoted as femtocell density. Therefore, with increasing FBS grid count, an increasing number of users are covered by FBSs, while the total number of users is kept 


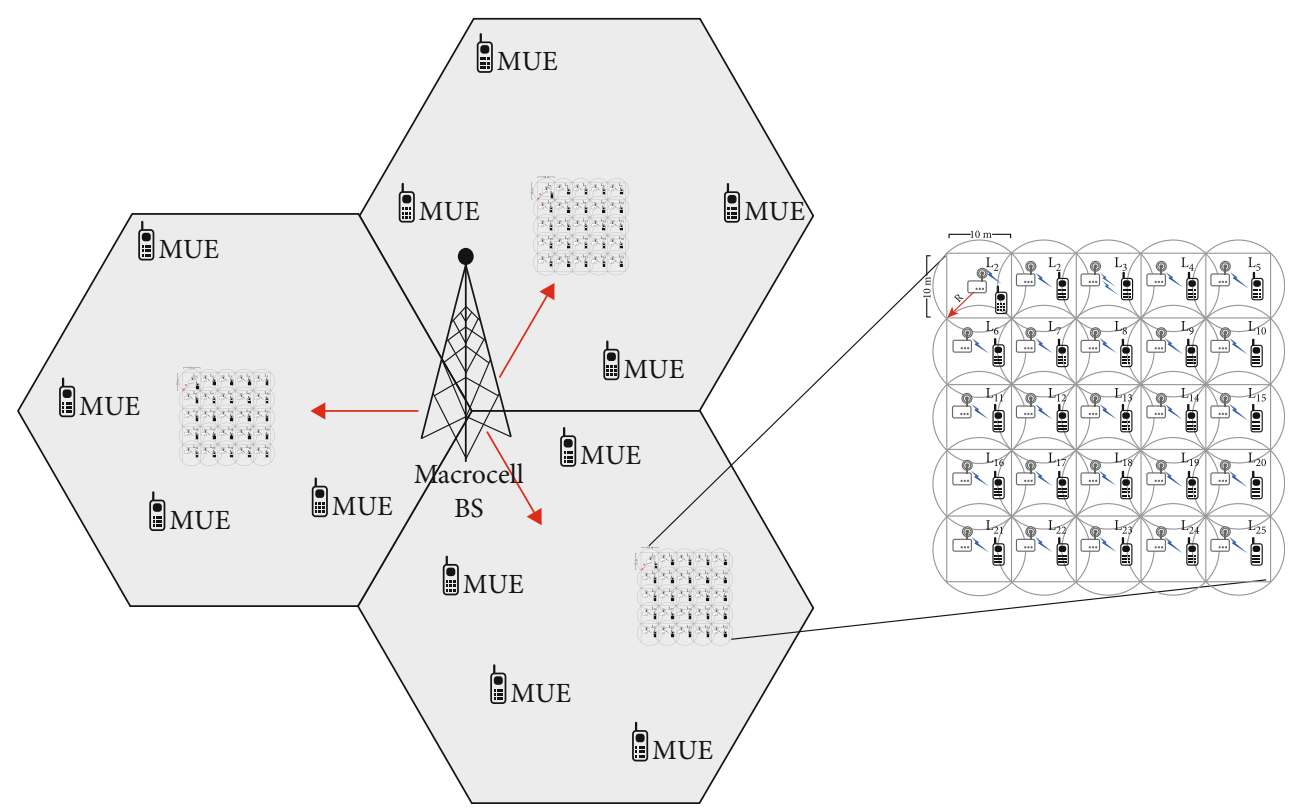

Figure 9: Two-tier HetNets for macrocell overlaid with femtocell grids $(5 \times 5)$.

constant. In simulation setup, FBSs operate in closed subscriber group (CSG) access mode which means only users of the corresponding indoor area are allowed to attach to the FBS, while users in the close vicinity of the femtocell experience strong interference. In the proposed system model simulation setup, the femtocell grid deployment either with size $(3 \times 3)$ or with size $(5 \times 5)$ will be randomly inside each macrocell sector area without any prior planning or coordination by either the user or the operator. The femtocell networks utilize from the same spectrum used by macrocell networks in what is called cochannel deployment. The cochannel deployment femtocells may reuse the same radio resources with macrocell and/or with the neighboring femtocells which resulting in cross-tier interference and cotier interference that decrease the quality of communication. So, under random densely deployments for femtocells, interference avoidance is considering the main challenge for network operators. Each MUE will be a victim of interference coming from neighboring femtocells and macrocell, if they use the same frequency subbands.

The impact of various femtocell grid deployment densities on downlink performance in two-tier HetNets will be investigated and evaluated using Vienna LTE system level simulator. Keep in mind that when both femtocell and macrocell using the same bandwidth in the same time, this will cause what is called cross-tier interference between macrocell and femtocell. In addition, from femtocell grid deployment models as illustrated in Figures 6(b) and 7(b), it is clear that some of the power and/or coverage of each FBS will be radiated outside the user premise. This radiated power will cause cotier interference between the neighboring femtocells in each FBS grid. Both co- and cross-tier interferences have a negative impact on degrading the performance of macrocell system. So, efficient resource allocation is indeed vital for interference avoidance is such systems. By adopting a suitable resource allocation technique, cotier interferences can be effectively avoided, while cross-tier interferences can be considerably minimized in order to improve the overall system throughput.

\section{Performance Evaluation Metrics}

The impact of various femtocell deployment densities on downlink performance in two-tier HetNet for femtocell will be investigated and evaluated using Vienna LTE system level simulator. The performance evaluation will be carried out in terms of signal-to-interference-and-noise ratio SINR, throughput, fairness, outage probability, and spectral efficiency.

5.1. Signal-to-Interference-Noise Ratio. In two-tier heterogeneous networks, some users will be under macrocell BS coverage while the remaining are covered by FBSs. If the user is covered by an FBS indoor area then, the achieved SINR of a femtocell-attached user can formally be written as:

$$
\operatorname{SINR}_{\mathrm{FUE}}=\frac{S_{\text {femto }}}{I_{\text {macro }}+I_{\text {femto }}+\text { Nois }},
$$

where $S_{\text {femto }}$ is the FBS signal and $I_{\text {macro }}$ and $I_{\text {femto }}$ are the interference from neighboring macro- and femtocell, respectively, that use the same subbands as the serving FBS.

The formula in (2) for achievable downlink SINR for any FUE served by FBS can be calculated by the formula

$$
\operatorname{SINR}_{\mathrm{FUE}}=\frac{P_{R}^{F}}{\sum_{b=1}^{N_{m}} \sum_{s=1}^{3} P_{R}^{m, s}+\sum_{\substack{f=1, f \neq F}}^{N_{f}} P_{R}^{f}+N_{o}},
$$

where $P_{R}^{F}$ is the serving FBS received power and $P_{R}^{m, s}$ and $P_{R}^{f}$ are the received power from interfering MBS and 
FBS, respectively, in current sector area that used the same frequency subbands as the base station that FUE attached with $P_{R}^{m, s} ; P_{R}^{f}$ will consider as zero if the corresponding cells use different frequency subbands. $N_{m}$ and $N_{f}$ are the number of MBSs and FBSs, respectively. $N_{m}$ may set to 1,7 , and 19 for one-tier or single macrocell (zero rings), one-tier/(one ring) macrocell, and two tiers (two-rings), respectively. That said, the three types will have zero ring, one ring, and two rings which produce the 1,7 , and 19 designs. $N_{o}$ is the noise power. The received power $P_{R}$ can directly calculate using the formula

$$
P_{R}(\mathrm{~dB})=P_{T}(\mathrm{~dB})-\mathrm{PL}_{\text {tot }}(\mathrm{dB})
$$

$P_{T}$ is the power transmitted from the serving base station in $\mathrm{dB}$. $\mathrm{PL}_{\text {tot }}$ is the total path loss experience by the signal in $\mathrm{dB}$ and can be expressed by the formula

$$
\mathrm{PL}_{\text {tot }}(\mathrm{dB})=\mathrm{PL}(\mathrm{dB})-G_{T}(\mathrm{~dB})
$$

where $\operatorname{PL}(\mathrm{dB})$ and $G_{T}(\mathrm{~dB})$ are the macroscopic path loss and gain of transmitting antenna in $\mathrm{dB}$, respectively.

5.1.1. Propagation Path Loss Models. Path loss model is depending on the propagation environment. The path loss calculation is depending on whether a signal originates from a MBS or a FBS; we choose between two propagation path loss models; outdoor path loss denoted as $\mathrm{PL}_{\text {outdoor }}$ and/or $\mathrm{PL}_{m}$ and indoor pathless which denoted as $\mathrm{PL}_{\text {indoor }}$ and/or $\mathrm{PL}_{f}$. Path loss model for macro base station users $\left(\mathrm{PL}_{m}\right)$ specified according to [43] subclause 4.5.2 is utilized and can be calculated by the formula

$$
\mathrm{PL}_{m}=28+35 \log _{10}(d) \mathrm{dB},
$$

where $d$ is the distance between user and macrocell base station in meters. Within the indoor area, the propagation path loss model for FBS users $\left(\mathrm{PL}_{f}\right)$ specified as in [44] subclause 5.24 is employed and can be calculated by formula

$$
\mathrm{PL}_{f}=38.5+20 \log _{10}\left(R_{i}\right)+L_{\text {walls }} \mathrm{dB} \text {, }
$$

where $R_{i}$ is the indoor distance between user and FBS in meters. The indoor area assumes to be isolated from the outdoor environment by a wall. At distance $R_{i}$, the signal is attenuated by the wall penetration loss, which denoted as $L_{\text {walls }} \cdot L_{\text {walls }}$ is explicitly modelled as

$$
\begin{gathered}
L_{\text {walls }} \mathrm{dB}=7 \mathrm{dBm}, 0<d \leq 10, \\
L_{\text {walls }} \mathrm{dB}=10 \mathrm{dBm}, 10<d \leq 20, \\
L_{\text {walls }} \mathrm{dB}=15 \mathrm{dBm}, 20<d \leq 30,
\end{gathered}
$$

(For light internal/internal/external walls, respectively, $3 \mathrm{~dB}$ for doors and $1 \mathrm{~dB}$ for window) As a transmitting antenna, the gain describes how well the antenna converts input power into radio waves headed in a specified direction. As a receiving antenna, the gain describes how well the antenna converts radio waves arriving from a specified direction into electrical power. Antenna gain is usually defined as the ratio of the power produced by the antenna from a far-field source on the antenna's beam axis to the power produced by a hypothetical lossless isotropic antenna, which is equally sensitive to signals from all directions. Usually, this ratio is expressed in decibels. A plot of the gain as a function of direction is called the radiation pattern. In the field of antenna design, the term radiation pattern (or antenna pattern or far-field pattern) refers to the directional (angular) dependence of the strength of the radio waves from the antenna or other source. The transmitting antenna radiation pattern is formulated as in [45]:

$$
G_{T}(\theta)=-\min \left[12\left(\frac{\theta}{\theta_{3 \mathrm{~dB}}}\right)^{2}, A m\right],-180^{\circ} \leq \theta \leq 180^{\circ}
$$

When considered $\theta_{3 \mathrm{~dB}}=65^{\circ}$ for $3 \mathrm{~dB}$ beam width and $A m=20 \mathrm{~dB}$ as maximum attenuation, equation (8) can be simplified to

$$
G_{T}(\theta)=-\min \left[12\left(\frac{\theta}{65^{\circ}}\right)^{2}, 20\right],-180^{\circ} \leq \theta \leq 180^{\circ} .
$$

Similarly, if the MUE is located under outdoor MBS coverage or located indoor but not coverage by FBS, then it will be considered as outdoor MUE and can communicate with the nearest MBS in such case; MUE is a victim for interference originates from all MBSs and FBSs in the vicinity that are using the same subbands which assigned to the serving MBS. The achievable SINR for any MUE can be expressed as

$$
\operatorname{SINR}_{\mathrm{MUE}}=\frac{S_{\text {macro }}}{I_{\text {macro }}+I_{\text {femto }}+\text { Nois }},
$$

where $S_{\text {macro }}$ is the MBS signal and $I_{\text {macro }}$ and $I_{\text {femto }}$ are the interference from neighboring macro- and femtocell, respectively, that use the (same subbands). If the indoor area is not covered by an FBS, then users are attached to the MBS, and the formula in (10) of the downlink SINR for any MUE served by sector area $S$ of MBS can be calculated as

$$
\operatorname{SINR}_{\mathrm{MUE}}=\frac{P_{R}^{M, S}}{\sum_{\substack{\boldsymbol{s}=1 \\ \boldsymbol{N} \neq S}}^{N_{m}} \sum_{R}^{m, s}+\sum_{f=1}^{N_{f}} P_{R}^{f}+N_{o}},
$$

where $P_{R}^{M, S}$ is the serving MBS received power and $P_{R}^{m, s}$ and $P_{R}^{f}$ are the received power from interfering MBS and FBS, respectively, in current sector area that used the same frequency subbands as the base station that MUE attached with $P_{R}^{m, s} ; P_{R}^{f}$ will consider as zero if the corresponding cells use different frequency subbands.

$N_{m}$ and $N_{f}$ are the number of MBSs and FBSs, respectively. $N_{m}$ may set to 1,7 , and 19 for one tier or single macrocell, two-tier/(two rings) macrocell, and three tiers (three rings), respectively. $N_{o}$ is the noise power. 
5.2. Outage Probability (SINR Distribution). The outage probability metric is used to measure the QoS for different densities for femtocell grids deployment. Outage probability is defined as the probability of UEs (MUEs + FUEs) having SINR values less than a predefined $\operatorname{SINR}_{\text {threshold }} \gamma$, and it is given by the formula.

$$
\mathbb{P}(\text { outage })=\mathbb{p}\left(\operatorname{SINR}_{\mathrm{MUE} / \mathrm{FUE}}<\gamma\right)
$$

Channel Quality Indicator (CQI) is reflected by user SINR. The serving BS receiving the CQI values as a feedback from a user and according to these values, BS defines the suitable Modulation and Coding Scheme (MCS) that will be used in LTE system. The MCS in its turn decides the QoS and transmission rate in the network.

5.3. Throughput Performance. The throughput is the actual data transfer rate after interference is considered. In other words, it is the actual achievable capacity in (Mbps) for any user in the network, regardless that this user is MUE or FUE can be expressed by Shannon's Law as

$$
T_{\mathrm{MUE} / \mathrm{FUE}}=W_{i} \log _{2}\left(1+\mathrm{SINR}_{\mathrm{MUE} / \mathrm{FUE}}\right),
$$

where $W$ is total allocated bandwidth of subcarriers given to user $i, i \in, N$. Express the total number of all mobile users in the network for both macrocell and femtocells $\left(N_{\text {MUE }}+N_{\text {FUE }}\right)$. The downlink interference between user $i$ (FUE or MUE) and his selected BS (FBS or/MBS) can be measured using signal-to-interference-and-noise ratio SINR $_{\text {FUE/MUE }}$ which can be calculated by equations (2) and (11), respectively. The total throughput for all MUEs in the network can be calculated by the formula

$$
T_{\mathrm{Tot}_{\mathrm{MUE}}}=\sum_{i=1}^{N_{\mathrm{MUE}}} W \log _{2}\left(1+\operatorname{SINR}_{\mathrm{MUE}_{i}}\right) .
$$

Similarly, the total throughput for all FUEs in the networks can be calculated using the formula

$$
T_{\mathrm{Tot}, \mathrm{FUE}_{i}}=\sum_{i=1}^{N_{\mathrm{FUE}}} W \log _{2}\left(1+\operatorname{SINR}_{\mathrm{FUE}_{i}}\right) .
$$

Average throughput of MUEs and average throughput of FUEs are calculated by formulas

$$
\begin{gathered}
T_{\mathrm{avg}}^{M}=\frac{T_{\mathrm{Tot}, \mathrm{MUE}_{i}}}{N_{\mathrm{MUE}}}, \\
T_{\mathrm{avg}}^{F}=\frac{T_{\mathrm{Tot}_{\mathrm{FUE}}}}{N_{\mathrm{FUE}}} .
\end{gathered}
$$

The average throughput of all MUEs and FUEs is calculated for all their possible locations. Then, the overall average UE capacity can be calculated using equation (17).

$$
T_{\text {overall }}=\frac{N_{\mathrm{MUE}} T_{\mathrm{avg}}^{M}+N_{\mathrm{FUE}} T_{\mathrm{avg}}^{F}}{N_{\mathrm{MUE}}+N_{\mathrm{FUE}}},
$$

where $N_{M U E}$ represents the number of MUEs, $N_{\mathrm{FUE}}$ represents the number of FUEs, and $C_{\mathrm{avg}}^{M}$ and $C_{\text {avg }}^{F}$ are, respectively, the average of MUE and FUE throughputs. The average throughput of the network can be expressed using the overall average capacity of UEs. In order to evaluate the throughput performance while changing the number of femtocell grids, this approach has to be repeated.

5.4. Fairness Performance. Fairness shows how equally a resource is distributed over $N$ users. The fairness function is applied in any system which share a resource. The imbalance between macrocell and femtocell users is quantified by the widely used Jain's Fairness Index (JFI) which formulated as [46].

$$
f(T)=\frac{\left(\sum_{i=1}^{N} T_{i}\right)^{2}}{N \sum_{i=1}^{N} T_{i}^{2}},
$$

where $N$ is the total number of users (MUEs + FUEs) in the network and $T_{i}$ denotes a vector of length $(N)$ which is containing the throughput achieved by each user $i$ of the $N$ users. Fairness index always lies between 0 and 1 (i.e., a resource distribution algorithm with fairness of 0.1 means that it is unfair for $90 \%$ of the users). Fairness is lowest for a small number of femtocells and increases with higher femtocell density.

5.5. Spectral Efficiency Performance. Spectrum or spectral efficiency (SE) is a measure how a limited frequency spectrum is used by the physical layer protocol. It sometime called bandwidth efficiency since it refers to the information rate which can be transferred over a given bandwidth in a cellular system. Spectrum efficiency is usually measured in (bit/s)/Hz per cell or in (bit/s)/Hz per unit area or in (bit/s)/Hz per site. SE is expressed as the average of bits per second successfully received by the UE device per unit spectrum. The average network spectral efficiency, from (13) and (14) SE, is thus given by equation (19):

$$
\mathrm{SE}=\frac{T_{\mathrm{Tot}, \mathrm{MUE}_{i}}+T_{\mathrm{Tot}_{\mathrm{FUE}}}}{B_{\text {tot }}},
$$

where $T_{\text {Tot,MUE }_{i}}$ and $T_{\text {Tot,FUE }_{i}}$ are the total throughput for all MUEs and total throughput for all FUEs in the network $B_{\text {tot }}$, which is the total bandwidth. 
TABLE 4: Macrocell simulation parameters.

\begin{tabular}{|c|c|}
\hline Parameter & Value \\
\hline Channel bandwidth & $20 \mathrm{MHz}$ \\
\hline Centre frequency & $2.14 \mathrm{GHz}$ \\
\hline Number of subchannels & 100 \\
\hline Number of occupied subcarriers & 1200 subcarriers \\
\hline Subcarrier spacing & $15 \mathrm{kHz}$ \\
\hline No. of macrocells & 7 cell sites ( 3 sectors per cell) \\
\hline Macrocell inter BS distance coverage & $500 \mathrm{~m}$ \\
\hline Tx X Rx antennas & $1 \times 1$ \\
\hline Macrocell BS max. Tx power & $20 \mathrm{w}$ or $/ 46 \mathrm{dBm} /$ or $49 \mathrm{~dB}$ recommended for $20 \mathrm{MHz}$ \\
\hline Macro BS antenna pattern & $\begin{array}{l}\text { Sectored directional antenna (3 sectors) } \\
\qquad G_{T}(\theta)=-\min \left[12\left(\frac{\theta}{65^{\circ}}\right)^{2}, 20\right]\end{array}$ \\
\hline No. of MUEs per macrosector & $40-60$ \\
\hline MU min-distance from macrocell & $25 \mathrm{~m}$ \\
\hline Macro minimum coupling loss & $70 \mathrm{dBm}$ \\
\hline White noise power spectral density & $-174 \mathrm{dBm} / \mathrm{Hz}$ \\
\hline Channel model: Rayleigh fading, path loss & $\mathrm{PL}_{\text {outdoor }}=28+35 \log _{10}(d) \mathrm{dB}$ \\
\hline Outdoor to indoor wall penetration loss $\left(L_{\mathrm{OI}}\right)$ & $\begin{array}{c}L_{\text {walls }} \mathrm{dB}=7 \mathrm{dBm}, 0<d \leq 10 \\
L_{\text {walls }} \mathrm{dB}=10 \mathrm{dBm}, 10<d \leq 20 \\
L_{\text {walls }} \mathrm{dB}=15 \mathrm{dBm}, 20<d \leq 30\end{array}$ \\
\hline Outdoor path loss exponent & 4 \\
\hline Shadow fading & $8 \mathrm{~dB}$ \\
\hline Resource allocation scheduling & Proportional fair \\
\hline Simulation time_TTI & 10 \\
\hline
\end{tabular}

TABLE 5: Femtocell specific simulation parameters.

\begin{tabular}{lc}
\hline Parameter & Value \\
\hline No. of FBS per macrocell sectors & $90($ FBS $3 \times 3)$ or $250($ FBS $5 \times 5)$ \\
FBS coverage (indoor area radius) & $20 \mathrm{~m}$ \\
Max. TX power of FBS & $20 \mathrm{~mW}(20 \mathrm{dBm})$ \\
Femtocell grid size & $(3 \times 3) /(5 \times 5)$ \\
No. of FBS grids & $1-10$ \\
FBS grid location inside macrocell sector area & Centre/edge/random \\
Location of FBS inside each house & Random/center \\
Number of UEs per FBS & $4-8$ \\
FBS access mode & CSG/OSG \\
Tx X Rx (1 $\times 1)$ FBS antenna pattern & Omnidirectional \\
Femto minimum coupling loss & $45 \mathrm{dBm}$ \\
Indoor path loss & \\
Indoor PL exponent & PL $_{\text {indoor }}=38.5+20 \log _{10}\left(R_{i}\right)+L_{\text {walls }} \mathrm{dB}$ \\
Femtocell resource allocation scheduling & 2 \\
Femto backhaul & Proportional fair
\end{tabular}

\section{Simulation Environment and Parameter Setup}

In this paper, the Vienna LTE system level simulator is developed in order to study the effects of various femtocell deployment densities on downlink performance of two-tier
HetNets [47]. In order to increase the density of femtocells in simulation test, the number of deployed FBS grids varies from 1 to 10 and from 1 to 7 for both FBS grids with sizes $(3 \times 3)$ and $(5 \times 5)$, respectively. The simulation platform allows the user to configure the number, type $(3 \times 3-5 \times 5)$, and position (center or edge) for all deployed FBS grids 


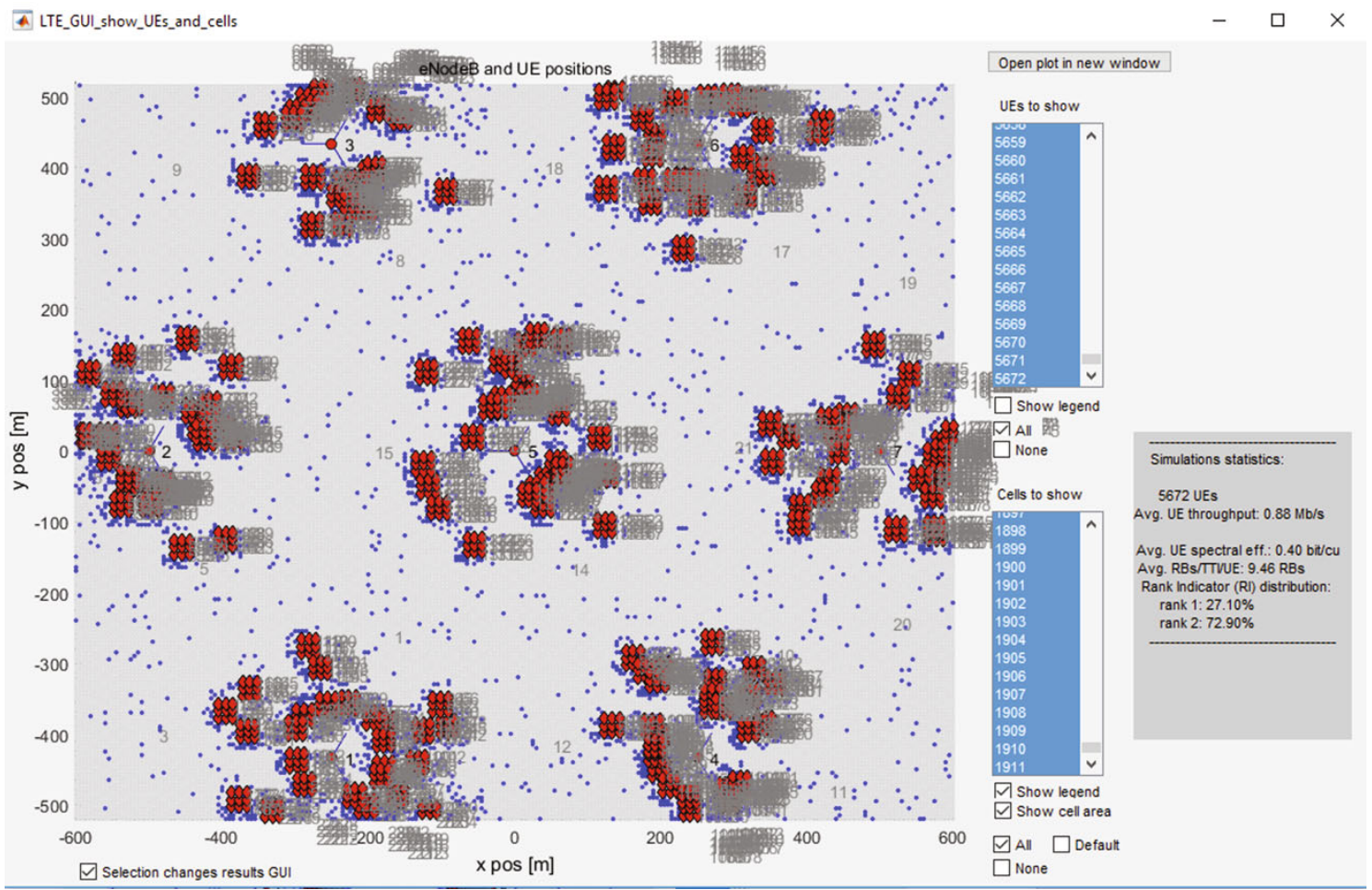

FIgURE 10: 10 FBS grid $(3 \times 3)$ deployments per macrocell sector area.

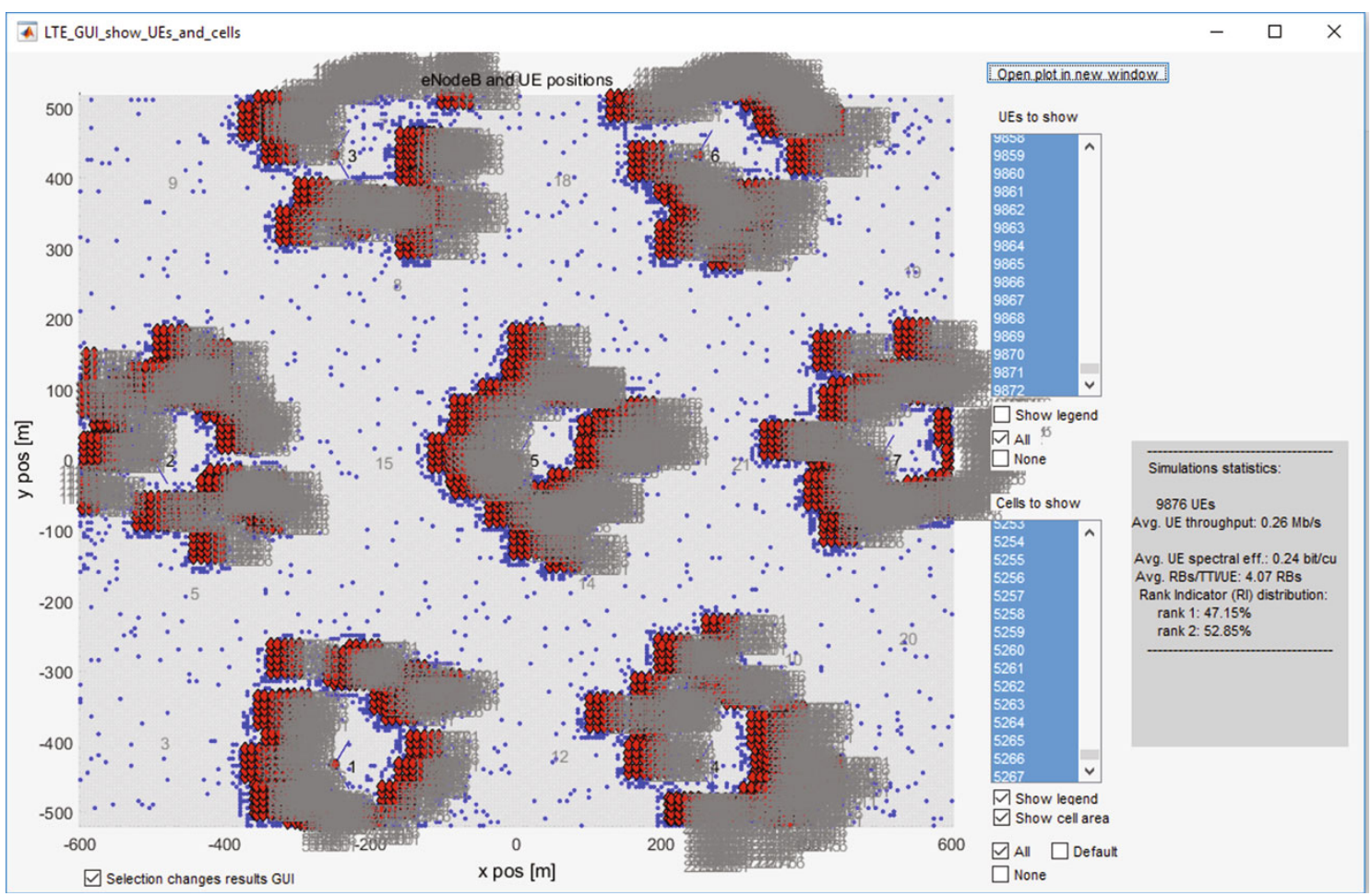

FIgURE 11: 10 FBS grid $(5 \times 5)$ deployments per macrocell sector area.

per macrocell sector. The proposed model for FBS grid deployment is implemented that the performance of the result was evaluated using the developed Vienna LTE system level simulator. The simulation parameters considered for both macrocell and femtocells are listed in Tables 4 and 5, respectively.

Figures 10 and 11 present a GUI screen shot for the simulation that show UEs, cells, and brief of simulation statistics 
when deploying two types of FBS grids $(3 \times 3)$ and $(5 \times 5)$, respectively.

\section{Simulation Results}

In this section, the simulation results and evaluation of performance are presented. The results show the effect of highdensity femtocell deployment in downlink performance for both macro- and femtocell network. The performance evaluation is carried out in terms of some measurement metrics such as signal-to-interference-and-noise ratio (SINR) distribution (outage probability), average user throughput, outage, and fairness index and/or user average spectral efficiency.

7.1. Outage Probability. Outage probability (SINR distribution) is the probability of SINR of random user less than a minimum threshold. In other words, a given user is considered in outage probability if the SINR of the link between the user and serving BS is not enough for a successful connection. Figure 12 depicts the SINR distribution of various densities for FBS grid deployments. The FBS deployment with various grid densities of $(3 \times 3)$ and $(5 \times 5)$ also shows the distribution when increasing the number of femtocells and/or FBS grids. In Figure 12, there are two references curves; the leftmost line depicts the distribution of macrocell only without FBSs, and the rightmost line shows the SINR distribution for high density FBS which is 5 grids of FBS with $(5 \times 5)$ size deployed per macrocell sector. In case of macrocell only, all users are communicated with macrocell BSs, and the interference only originates from the surrounding MBSs in the vicinity of central MBS. The other curves from left to right show the SINR distribution when the number of FBS grids per macrocell sector area increases; two types of interference occurred which are cross-tier interference between macrocell BS and FBS, in addition to the cotier interference among neighboring FBSs located in the same FBS grid. The lines in between the left most curve and rightmost curve represent the SINR distribution for various FBS grid densities per macrocell sector area which are one grid $(3 \times 3)$ or $9 \mathrm{FBSs}$, two grids $(3 \times 3)$ or $18 \mathrm{FBSs}$, one grid $(5 \times 5)$ or $25 \mathrm{FBSs}$, three grids $(3 \times 3)$ or $27 \mathrm{FBSs}$, four grids $(3 \times 3)$ or $36 \mathrm{FBSs}$, five grids $(3 \times 3)$ or $45 \mathrm{FBSs}$, two grids $(5 \times 5)$ or 25 FBSs, three grids $(5 \times 5)$ or 75 FBSs, four grids $(5 \times 5)$ or $100 \mathrm{FBSs}$, and five grids $(5 \times 5)$ or $125 \mathrm{FBSs}$, respectively. It is clear from the graph that by increasing the number of FBS, the SINR distribution inclined to the rightmost curve which represents the reference for high density FBS. This behavior of curves shows clearly that there is a big gap in the performance of MUEs and FUEs. Thus, in terms of SINR, it is of benefit to increase the number of FBS accompanied by a suitable effective interference management technique.

7.2. Average User Throughput. In this section, the impact of signal-to-interference-and-noise ratio on the throughput has been investigated. The throughput is evaluated for deployment of various FBS grid density and compared with the throughput with macrocell without any femtocell deployment and with macrocell enhanced network with femtocell added one by one. When the number of FBS grids per macrocell sector area increases, the overall aggregate average throughput (for both MUEs and FUEs) also increases monotonically and reaches its maximum when all indoor area is covered by FBS. In contrast, the simulation results show that both the average user throughput and overall average throughput decrease with high density deployment of FBSs because of severe interference and large number of users. Figures 13 and 14 depict the average user throughput $(\mathrm{Mb} / \mathrm{s})$ plotted versus various FBS grid densities. Although femtocell deployment as 2nd overlaid tier in the existing macrocell will improve the overall performance (i.e., throughput overall/throughput macrocellonly $>1$ ), the resource that offloading from the macro BS can mostly compensate for the harm caused by additional interference (i.e., throughput ${ }^{\text {macrocellonly/ }}$ throughput ${ }^{\text {overall }}<1$ ). The curves of macrocell only without FBSs on both Figures 13 and 14 are considered as reference. The simulation results show and compare the average user throughput for MUEs only (with no FBS grid deployment); overall average throughput for both MUEs and FUEs when increase FBS grid deployments with both sizes $(3 \times 3)$ and $(5 \times 5)$, the results are compared with overall average throughput for both MUs and FUs when deployment equivalent number of femtocells one by one to the FBS grids $(3 \times 3)$ and $(5 \times 5)$ as shown in Figures 13 and 14 , respectively. The average throughput for FUEs only increases when deploy the 1 st and 2 nd FBS grids $(3 \times 3)$, while after that no significant change has occurred in FUE throughput, as shown clearly in Figure 13. On the other hand, when deploying FBS as grid of size $(5 \times 5)$, the average throughput for FUE only increases when adding 1st, 2nd, 3 rd, 4th, and 5th; then, when adding FBS grid numbers 6th and 7 th, the average throughput of FUEs slowly drops down as shown in Figure 14. The throughput degradation is due to the severe interference between macrocell and femtocell, in addition also to the interference between neighboring femtocells in the deployed FBS grids either with size $(3 \times 3)$ or $(5 \times 5)$. In contrast, both Figures 13 and 14 show when deployment FBS randomly one by one per macrocell sector area, the overall average throughput for both MUE and FUE will steadily improve when increasing the femtocell density, whereas the macrocell user's performance is downgraded severely as a result of cross-tier interference between macrocell and femtocells.

However, performance evaluation in terms of overall average throughput can be misleading, since the value is not achieved by any user, as seen in Figure 14 .

Figure 15 illustrates the scattering of individual user throughput versus SINR for a femtocell density when deploying 10 FBSs one by one and total of 80 MUEs per macrocell sector. The user throughput values are either much higher or much lower than the overall aggregate average throughput of $22.5 \mathrm{Mbit} / \mathrm{s}$, as seen in the first group (red dots in the upper right corner) which are identified as FBS attached users (FUEs) and second group (blue dots in the lower left corner) which are identified as macro BS attached users (MUEs), respectively.

A zoom out views only to blue dots that represent macrocell user's throughput when deployment 1 FBS grid 


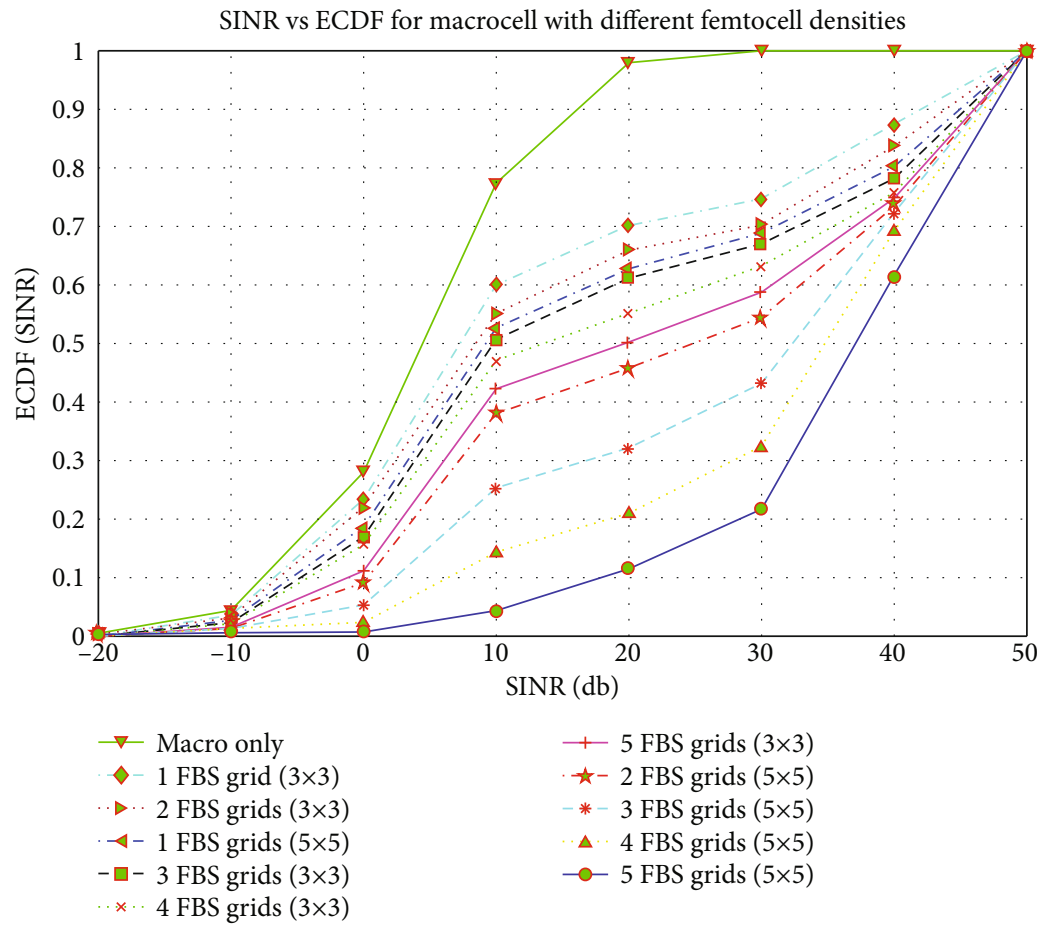

FIgURE 12: Outage probability for macrocell with various femtocell densities.

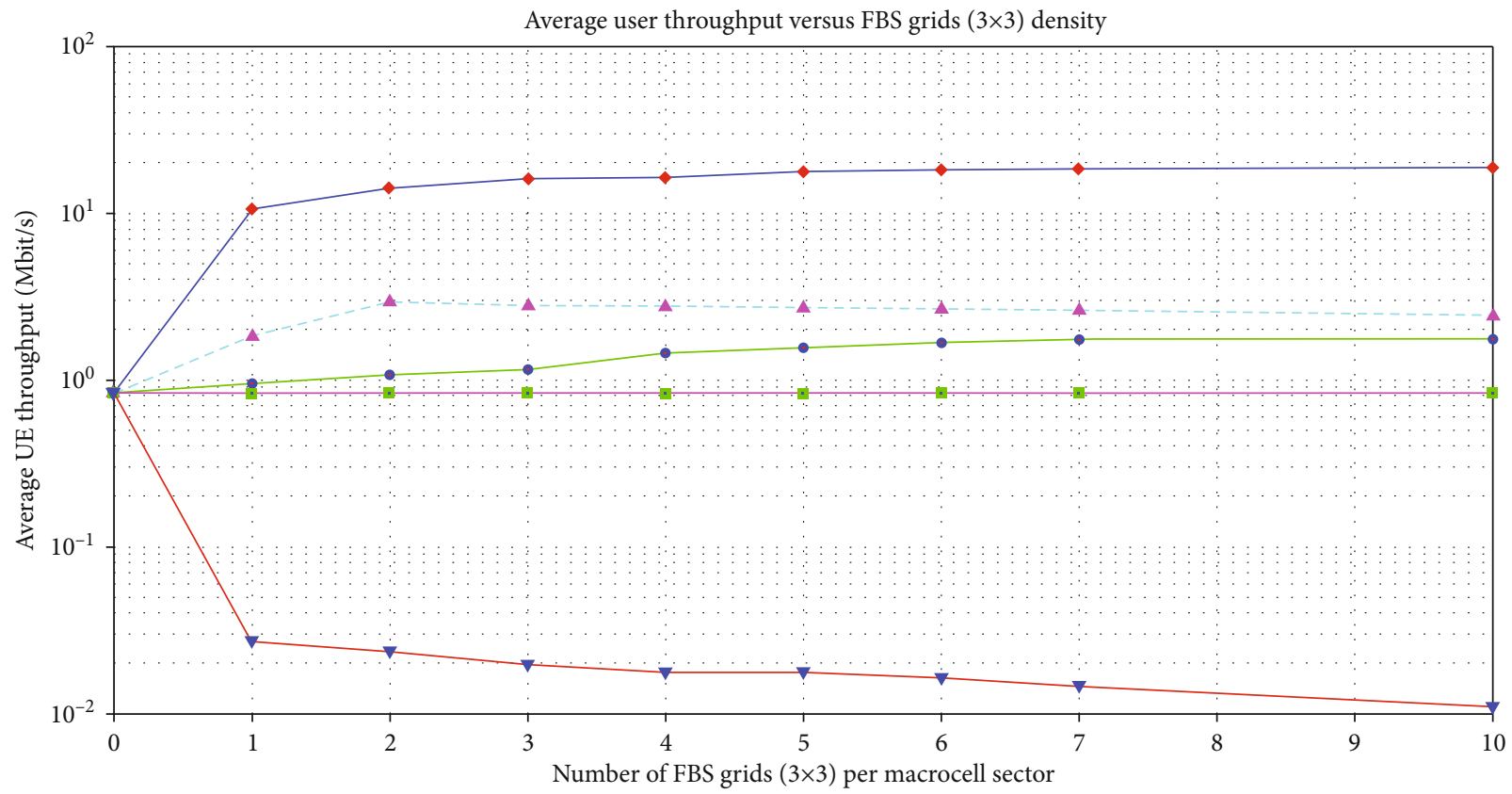

- Overall avg. throughput for both MU and FU when deploy equivalent no. of FBS to no. of FBS $(3 \times 3)$ grids

- Avg. FUE throughput when deployment FBS as grids $(3 \times 3)$

- Overall avg. throughput for both MU and FU when deployment FBS as grids $(3 \times 3)$

$\rightarrow-$ Avg MUE throughput (no femtocell deployment)

$\rightarrow$ Avg. throughput for only MUE when deployment FBS as grids $(3 \times 3)$

FIgURE 13: Average user throughput for various FBS grid $(3 \times 3)$ density. 


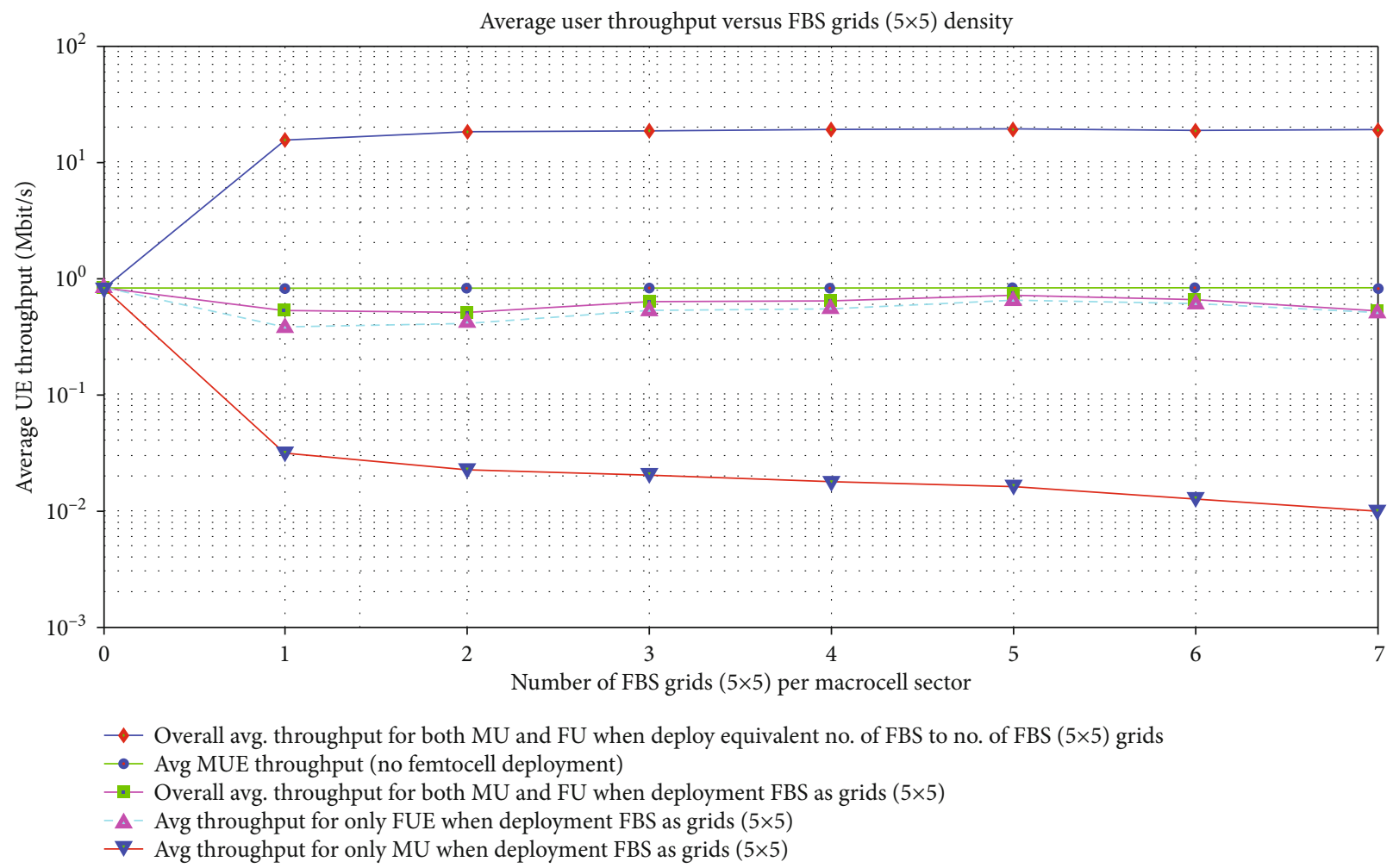

FIGURE 14: Average user throughput for various FBS grid $(5 \times 5)$ density.

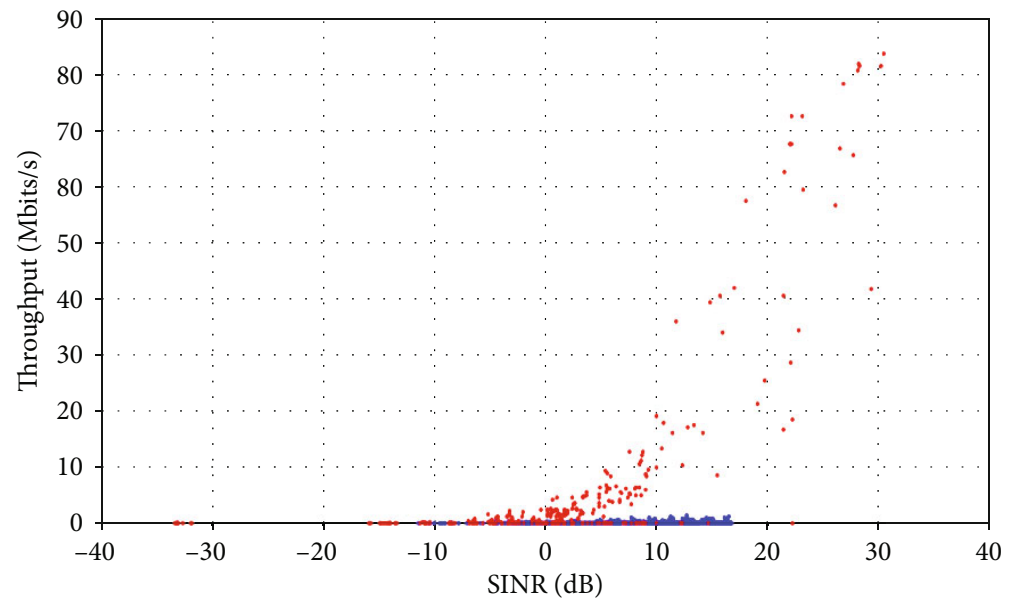

- Macro users

- Femto users

FIgURe 15: individual throughput of MUE vs. FUE when deploy 1 FBS grid $(3 \times 3)$.

$(3 \times 3)$. The results in Figure 16 show MUE throughput degradation because of the severe interference between macrocell and femtocell.

Figures 16 and 17 represent the scattering of individual user throughput versus SINR for a femtocell density when deployment FBS as grids of $(3 \times 3)$ and $(5 \times 5)$, respectively. The blue dots with low values in both figures represent the MUE throughput while the red dots represent the FUE throughput. The simulation results show the effects of cross-tier and cotier interference due to increasing density of FBSs; this effectiveness will be translated as degradation in macrocell user performance. As shown in Figure 18, the imbalance between macrocell and femtocell users was quantified by the widely used Jain's Fairness Index (JFI).

7.3. Fairness Index. Figure 19 portrays the fairness versus various densities of FBSs. The fairness index is low with lowest FBS density, and fairness monotonically increases with higher FBS density and reaches its maximum at a full femtocell coverage (i.e., all indoor areas are covered by FBS) as depicted in Figure 19 when deploying FBS randomly one by one inside macrocell sector area. However, when 


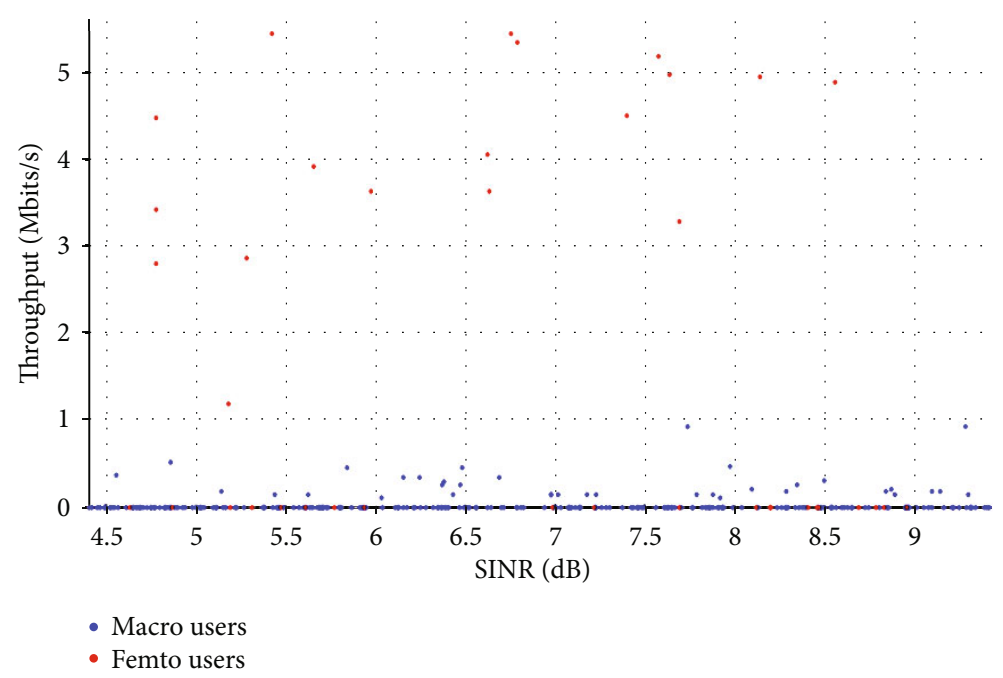

FIGURE 16: Individual throughput of only MUEs when deploy 1 FBS grid $(3 \times 3)$.

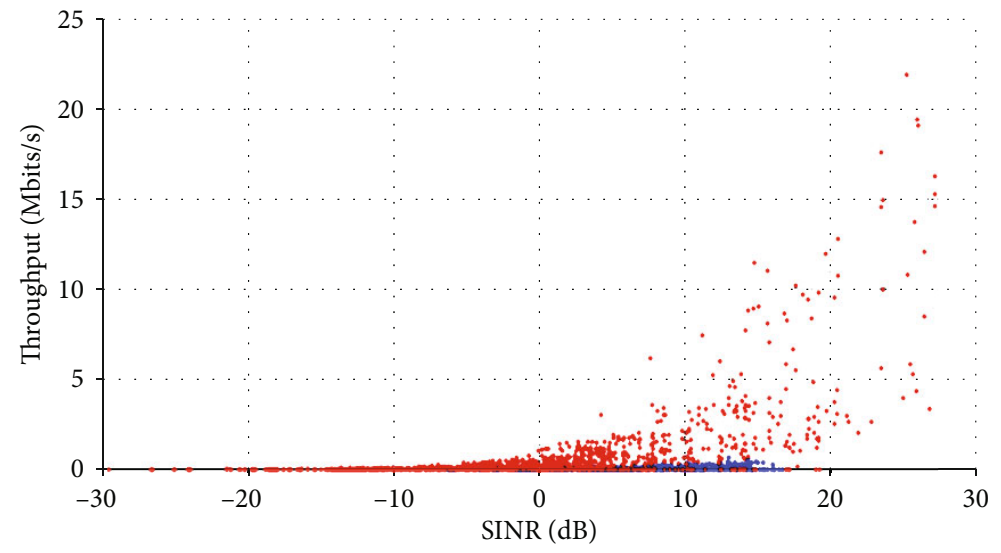

- Macro users

- Femto users

FIgURE 17: Individual throughput of MUE vs. FUE when deploy 1 FBS grid $(5 \times 5)$.

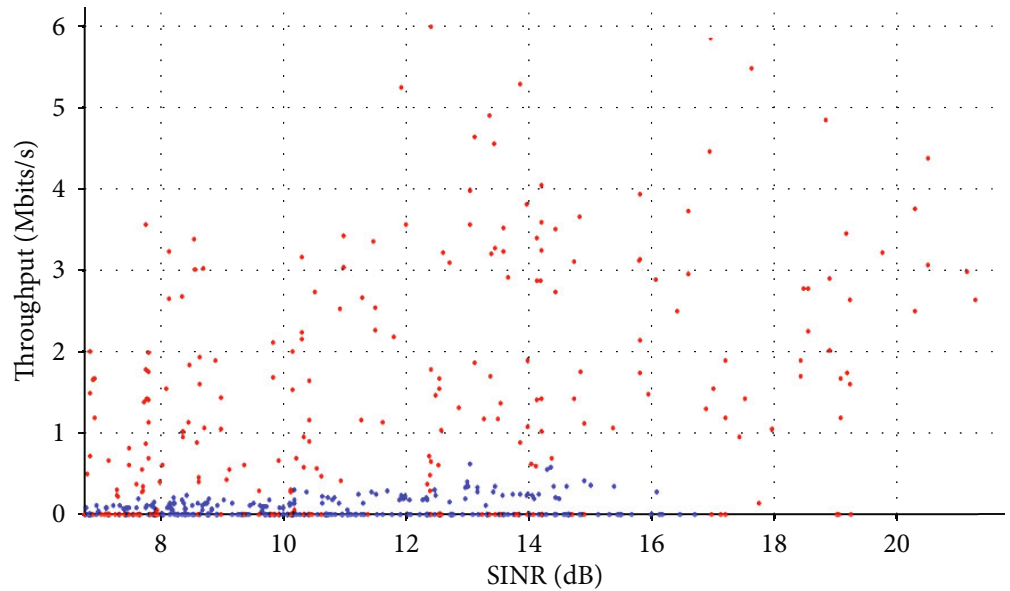

- Macro users

- Femto users

FIGURE 18: Macrocell user performance degradation. 


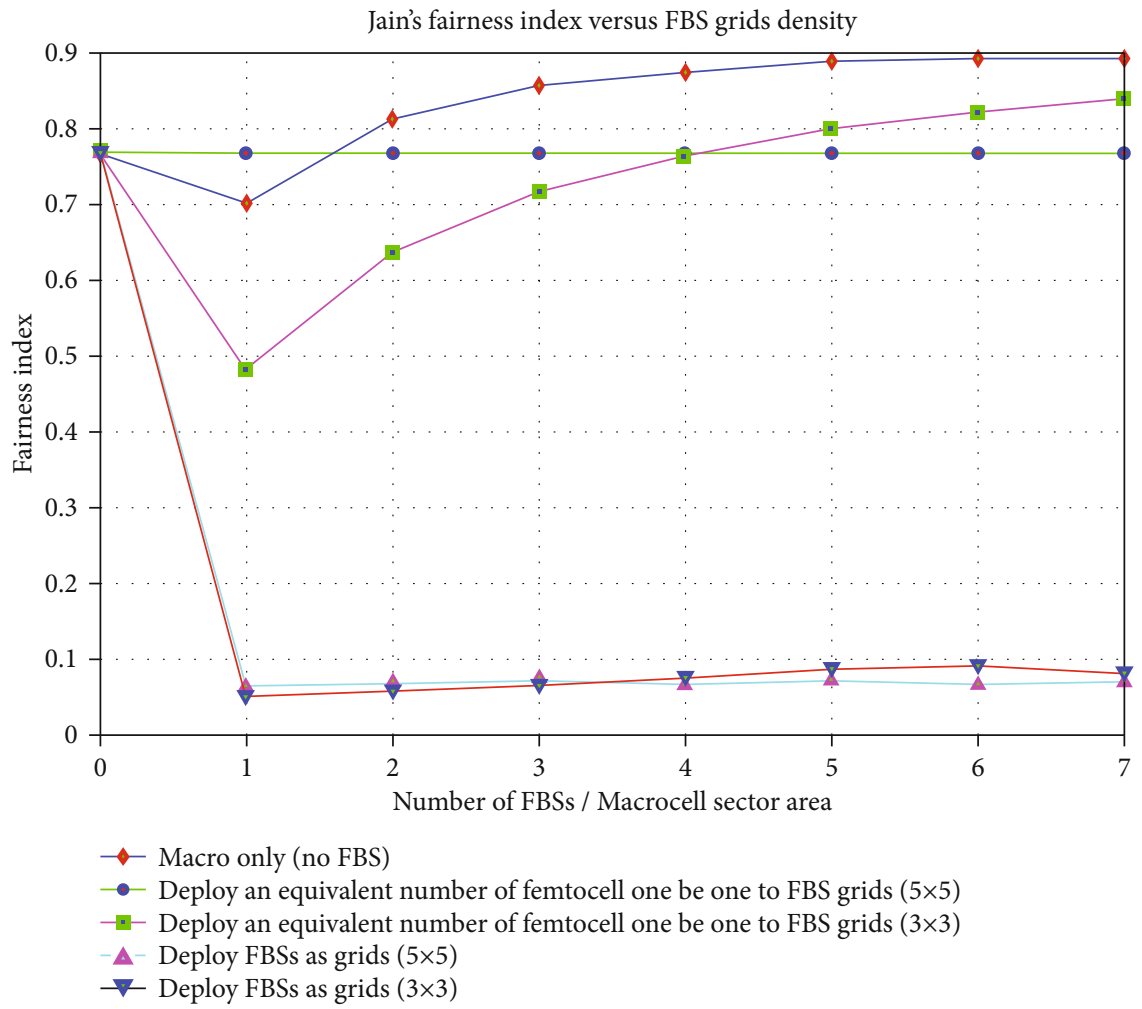

FIGURE 19: Jain's Fairness Index vs. the number of FBS grids.

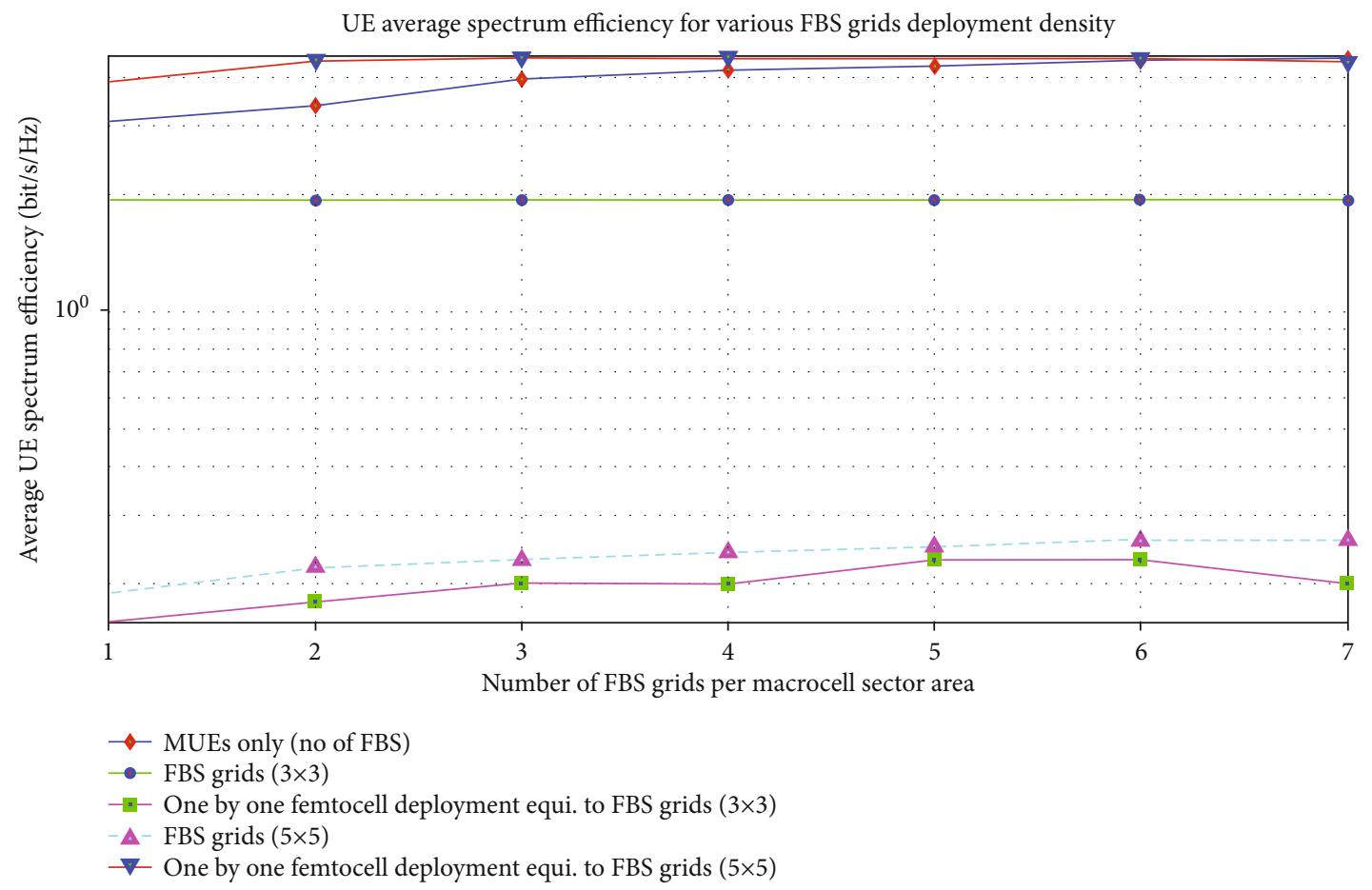

FIGURE 20: UE average spectrum efficiency vs. the number of FBS grids.

deployment the FBS as grids of both types $(3 \times 3)$ and $(5 \times 5$ ), the fairness index is drop down comparing to the equivalent deployment of FBSs one by one. The results show the fairness index when deploy FBS as grid $(3 \times 3)$ increases slowly with increasing the number of FBS grids $(3 \times 3)$ whereas there is no significant increase in fairness index when deployment FBSs as grids of size $(5 \times 5)$ as shown in Figure 19, the red continues curve and cyan discrete line, 
respectively. According to the type of resource allocation scheme, there is a trade-off between the fairness and average throughput; in other words, some resource allocation schemes can achieve high fairness in the expense of throughput. In contrast, some other resource allocation schemes can provide a higher throughput in the expense of fairness. Due to imbalance between fairness and throughput, innovated interference management techniques based optimal resource allocation are needed in order to achieve high throughput and acceptable fairness level. The fairness of high-density scenario (i.e., $5 \times 5$ grid of FBS) highly decreases due to the unmanaged cross-tier interference between macrocell BSs and FBSs as well as cotier interference among neighboring FBSs.

7.4. UE Average Spectral Efficiency. Figure 20 shows the spectrum efficiency versus the number of deployed FBS grids. The results of spectral efficiency for both $(3 \times 3)$ and $(5 \times 5)$ FBS grid deployments are compared with sum spectral efficiency for macrocell only without femtocell as a reference. The result graph shows that, when increase the number of FBS grids per macrocell sector area, the spectrum efficiency increases until the curve is flattened with a high density of FBS grid deployment. The increase of spectrum efficiency is due to offloading macro users to be femtocell users. In addition, increasing the number of FBS will increase the reuse of frequency. Whereas the flatten of the curves occurs because of the severe interference resulting from the increased number of FBS gird deployment inside macrocell sector area as clearly seen in Figure 20.

\section{Conclusion}

The pervasive increase of wireless devices in last years which are all connected to similar system makes managing and serving these devices highly challenging. The difficulty occurs in providing high data rates with very low latency and strong reliability to these devices. All that can be summarized as providing high-quality of wireless service. In this regard, femtocells are considered as the most effective promising small cell technology that can provide a high-quality coverage for indoor environments, as well as, to increase data rates, capacity, and efficiency for the $5 \mathrm{G}$ wireless communication cells that will be deployed as close as 10 meters away from each other, and by 2020, the number of wireless devices will reach up to 50 billion.

However, deploying huge number of femtocell networks without very tight network planning is a real challenge and may worsen the service instead of improving it. The main challenge in deploying dense femtocell networks is avoiding and minimizing interference between nearby cells. Interference mainly occurs since the radio resource is scarce, and new femtocells are randomly deployed and installed by users without any prior planning from the operator. Femtocells need to share the available spectrum (operating on the same licensed frequency band) with the existing macrocells in the network. This process is referred to as cochannel deployment. In cochannel deployment, the femtocells and macrocells can fully or partially share/reuse the available bandwidth, thereby resulting in severe cross-tier interferences between macrocells and femtocells and cotier interference among neighboring femtocells, respectively.

In this work, the proposed model of FBS grid deployment was implemented and integrated, and the performance of the impacts of various density of FBS grid deployment was evaluated with Vienna LTE system level simulator. The simulation results show that FBS grid deployment improves overall average user throughput in case of low and medium density scenario. However, for high density scenario, FBS grid deployment has no enhancement in terms of fairness and throughput. The results confirmed that when deploying the FBS grids inside macrocell sector, there is a trade-off; in one hand, this technology offloads the macrocell base station and increases the coverage and network capacity. In contrast, it brings an interference between macrocell and femtocell tiers (cotier interference) in addition to interference between neighboring femtocells (cotier interference); this interference will lead to degrade the overall performance of the network. Interferences happen exclusively in cases where both the aggressor and the victim attempted to use the same radio resources. Thus, efficient resource allocation is vital for interference avoidance in such systems. In addition, optimal interference mitigation techniques are needed to be developed not only in terms of enhancing overall user throughput but also to enhance the individual user performance by means of fairness.

\section{Data Availability}

None of that is investigated or related to our work.

\section{Conflicts of Interest}

The authors declare that they have no conflicts of interest.

\section{References}

[1] L. Polak, L. Klozar, O. Kaller, J. Sebesta, M. Slanina, and T. Kratochvil, "Study of coexistence between indoor LTE femtocell and outdoor-to-indoor DVB-T2-Lite reception in a shared frequency band," EURASIP Journal on Wireless Communications and Networking, vol. 2015, no. 1, 14 pages, 2015.

[2] S. Carlaw, IPR and the Potential Effect on Femtocell Markets, FemtoCells Europe, 2008.

[3] M. Ghanbarisabagh, G. Vetharatnam, E. Giacoumidis, and S. M. Malayer, "Capacity improvement in $5 \mathrm{G}$ networks using femtocell," Wireless Personal Communications, vol. 105, no. 3, pp. 1027-1038, 2019.

[4] S.-p. Yeh, S. Talwar, N. Himayat, and K. Johnsson, "Power control based interference mitigation in multi-tier networks," in 2010 IEEE Globecom Workshops, pp. 701-705, Miami, FL, USA, 2010.

[5] C.-J. Liu, P. Huang, L. Xiao, and A.-H. Esfahanian, "Inter-femtocell interference identification and resource management," IEEE Transactions on Mobile Computing, vol. 19, no. 1, pp. 116-129, 2019.

[6] A. Ghiamatyoun and A. Olfat, "Probability bounds of crosslayer interference in femtocell-underlay cellular networks 
employing interference alignment," IET Communications, vol. 13, no. 16, pp. 2590-2600, 2019.

[7] J. Cullen, Radioframe Presentation, Femtocell Europe, London, UK, 2008.

[8] L. T. Ho and H. Claussen, "Effects of user-deployed, cochannel femtocells on the call drop probability in a residential scenario," in 2007 IEEE 18th International Symposium on Personal, Indoor and Mobile Radio Communications, pp. 1-5, Athens, Greece, 2007.

[9] D. López-Pérez, G. de la Roche, A. Valcarce, A. Juttner, and J. Zhang, "Interference avoidance and dynamic frequency planning for WiMAX femtocells networks," in 2008 11th IEEE Singapore International Conference on Communication Systems, pp. 1579-1584, Guangzhou, China, 2008.

[10] M. Z. Chowdhury, Y. M. Jang, and Z. J. Haas, "Interference mitigation using dynamic frequency re-use for dense femtocell network architectures," in 2010 Second international conference on ubiquitous and future networks (ICUFN), Jeju, Korea (South), 2010.

[11] D. Chambers, Femtocell HistoryApril 2018, http://www .thinkfemtocell.com/FAQs/femtocell-history.html.

[12] V. Chandrasekhar, J. Andrews, and A. Gatherer, "Femtocell networks: a survey," IEEE Communications Magazine, vol. 46, no. 9, pp. 59-67, 2008.

[13] H. Claussen, L. T. Ho, and L. G. Samuel, "An overview of the femtocell concept," Bell Labs Technical Journal, vol. 13, no. 1, pp. 221-245, 2008.

[14] D. N. Knisely and F. Favichia, "Standardization of femtocells in 3GPP2," IEEE Communications Magazine, vol. 47, no. 9, pp. 76-82, 2009.

[15] WiMAX Forum, Requirements for WiMAX Femtocell Systems, 2009, Version 1.0.0.

[16] 3GPP Release 8http://www.3gpp.org/Release-8.

[17] D. N. Knisely, T. Yoshizawa, and F. Favichia, "Standardization of femtocells in 3GPP," IEEE Communications Magazine, vol. 47, no. 9, pp. 68-75, 2009.

[18] G. J. D. Harikumar, "Femtocells: implementation challenges and solutions," in Director, CDMA Femtocell program, Airvana Incorporated, IEEE Globecomm, 2008.

[19] S. Sesia, I. Toufik, and M. Baker, LTE-the UMTS Long Term Evolution: From Theory to Practice, John Wiley \& Sons, 2011.

[20] 3GPP Technical Specification TS 36213 V1030, Evolved Universal Terrestrial Radio Access (E-UTRA); Physical Layer Procedures, 2011, http://www.3gpp.org/ftp/Specs/html-info/ 36213.htm.

[21] T. Nakamura, S. Nagata, A. Benjebbour et al., "Trends in small cell enhancements in LTE advanced," IEEE Communications Magazine, vol. 51, no. 2, pp. 98-105, 2013.

[22] S. Xiao, X. Zhou, Y. Yuan-Wu, G. Y. Li, and W. Guo, "Robust resource allocation in full-duplex-enabled OFDMA femtocell networks," IEEE Transactions on Wireless Communications, vol. 16, no. 10, pp. 6382-6394, 2017.

[23] A. Khandekar, N. Bhushan, J. Tingfang, and V. Vanghi, "LTEadvanced: heterogeneous networks," in 2010 European wireless conference (EW), pp. 978-982, Lucca, Italy, 2010.

[24] M. Al-Omari, A. R. Ramli, A. Sali, and R. S. Azmir, "Deployment of femtocell and its interference management approaches in LTE heterogeneous networks," Journal of Theoretical and Applied Information Technology, vol. 87, no. 1, p. $54,2016$.
[25] L. Lindbom, R. Love, S. Krishnamurthy, C. Yao, N. Miki, and V. Chandrasekhar, "Enhanced inter-cell interference coordination for heterogeneous networks in lte-advanced: a survey," 2011, https://arxiv.org/abs/1112.1344.

[26] R. Bendlin, V. Chandrasekhar, R. Chen, A. Ekpenyong, and E. Onggosanusi, "From homogeneous to heterogeneous networks: a 3GPP long term evolution rel. 8/9 case study," in 2011 45th Annual Conference on Information Sciences and Systems, pp. 1-5, Baltimore, MD, USA, 2011.

[27] D. López-Pérez, A. Valcarce, G. De La Roche, and J. Zhang, "OFDMA femtocells: a roadmap on interference avoidance," IEEE Communications Magazine, vol. 47, no. 9, pp. 41-48, 2009.

[28] J. Ghosh and D. N. K. Jayakody, "Game theoretic frequency reuse approach in OFDMA femtocell networks," Transactions on Emerging Telecommunications Technologies, vol. 29, no. 10, p. e3440, 2018.

[29] S.-Y. Pyun, W. Lee, and O. Jo, "Uplink resource allocation for interference mitigation in two-tier femtocell networks," Mobile Information Systems, vol. 2018, 6 pages, 2018.

[30] G. A. Safdar, "Coverage area-based power control for interference management in LTE femtocells," in LTE Communications and Networks: Femtocells and Antenna Design Challenges, pp. 84-103, 2018.

[31] Z. Liu, Y. Yuan, H. Yuan, and X. Guan, "Power allocation based on proportional-integral controller in femtocell networks with consideration of maximum power constraint," IEEE Systems Journal, vol. 13, no. 1, pp. 88-97, 2018.

[32] M. Al-omari, A. R. Ramli, A. Sali, and R. S. Azmir, "A femtocell cross-tier interference mitigation technique in OFDMALTE system: a cuckoo search based approach," Indian Journal of Science and Technology, vol. 9, no. 2, 2016.

[33] A. Salman, I. M. Qureshi, S. Saleem, S. Saeed, and B. R. Alyaei, "Novel sensing and joint beam and null steering based resource allocation for cross-tier interference mitigation in cognitive femtocell networks," Wireless Networks, vol. 24, no. 6, pp. 2205-2219, 2018.

[34] N. Saquib, E. Hossain, L. B. Le, and D. I. Kim, "Interference management in OFDMA femtocell networks: issues and approaches," IEEE Wireless Communications, vol. 19, no. 3, pp. 86-95, 2012.

[35] K.-C. Ting, W. Lin, and C.-P. Wang, “A distributed power control scheme for the mitigation of co-tier downlink interference for femtocell in the future 5G networks," in International Conference on Internet of Things as a Service, pp. 73-85, Cham, 2017.

[36] T. Lee, J. Yoon, S. Lee, and J. Shin, "Interference management in OFDMA femtocell systems using fractional frequency reuse," in 2010 international conference on communications, circuits and systems (ICCCAS), pp. 176-180, Chengdu, China, 2010.

[37] K. C. Ting, W. Lin, and C. Wang, "Loading aware green power control (LAGPC) for the mitigation of co-tier downlink interference for femtocell in the future 5G networks," Mobile Networks and Applications, vol. 24, no. 3, pp. 864-877, 2019.

[38] E. S. Attia, S. A. El-Dolil, and M. Abd-Elnaby, "Spectrum allocation for enhanced cross-tier interference mitigation with throughput improvement for femtocells in a heterogeneous LTE cellular network," Wireless Personal Communications, vol. 101, no. 3, pp. 1671-1683, 2018.

[39] D. Calin, H. Claussen, and H. Uzunalioglu, "On femto deployment architectures and macrocell offloading benefits in joint 
macro-femto deployments," IEEE Communications Magazine, vol. 48, no. 1, pp. 26-32, 2010.

[40] J. Ling, D. Chizhik, and R. Valenzuela, "On resource allocation in dense femto-deployments," in Microwaves, Communications, Antennas and Electronics Systems, 2009. COMCAS 2009. IEEE International Conference on, pp. 1-6, Tel Aviv, Israel, 2009.

[41] Z. Sun, C. Yin, and G. Yue, "Reduced-complexity proportional fair scheduling for OFDMA systems," in 2006 International Conference on Communications, Circuits and Systems, vol. 2, pp. 1221-1225, Guilin, China, 2006.

[42] M. Haenggi and R. K. Ganti, "Interference in large wireless networks," Foundations Trends in Networking, vol. 3, no. 2, pp. 127-248, 2009.

[43] 3GPP, Evolved Universal Terrestrial Radio Access (EUTRA); Radio Frequency (RF) System Scenarios (Release 10), vol. TR 36.942, 3rd Generation Partnership Project (3GPP), 2010.

[44] Alcatel-Lucent, Simulation Assumptions and Parameters for FDD HeNB RF Requirements, 2009.

[45] K. Han, Y. Choi, D. Kim, M. Na, S. Choi, and K. Han, “Optimization of femtocell network configuration under interference constraints," in 2009 7th International Symposium on Modeling and Optimization in Mobile, Ad Hoc, and Wireless Networks, pp. 1-7, Seoul, Korea (South), 2009.

[46] R. K. Jain, D.-M. W. Chiu, and W. R. Hawe, A Quantitative Measure of Fairness and Discrimination, Eastern Research Laboratory, Digital Equipment Corporation, Hudson, MA, 1984.

[47] M. Rupp, S. Schwarz, and M. Taranetz, The Vienna LTEAdvanced Simulators, Springer, 2016. 